\title{
In vivo lentiviral vector gene therapy to cure hereditary tyrosinemia type 1 and prevent development of precancerous and cancerous lesions
}

Clara T Nicolas ${ }^{1,2,3}$, Caitlin J VanLith ${ }^{1}$, Kari L Allen ${ }^{1}$, Raymond D Hickey ${ }^{1,4}$, Zeji Du ${ }^{1}$, Lori G Hillin $^{1}$, Rebekah M Guthman ${ }^{1,5}$, William J Cao ${ }^{1}$, Aditya Bhagwate ${ }^{6}$, Daniel O’Brien ${ }^{6}$, JeanPierre Kocher $^{6}$, Robert A Kaiser ${ }^{1,7}$, Stephen J Russell ${ }^{4}$, Joseph B Lillegard ${ }^{1,7,8}$

1. Department of Surgery, Mayo Clinic, Rochester, MN

2. Faculty of Medicine, University of Barcelona, Barcelona, Spain

3. Department of Surgery, University of Alabama Birmingham, Birmingham, AL

4. Department of Molecular Medicine, Mayo Clinic, Rochester, MN

5. Medical College of Wisconsin, Wausau, WI

6. Department of Biomedical Statistics and Informatics, Mayo Clinic, Rochester, MN

7. Midwest Fetal Care Center, Children's Hospitals and Clinics of Minnesota, Minneapolis, MN

8. Pediatric Surgical Associates, Minneapolis, MN

Conflict of Interest Statement: The authors have declared that no conflict of interest exists. 


\begin{abstract}
Conventional therapy for hereditary tyrosinemia type-1 (HT1) with 2-(2-nitro-4-

trifluoromethylbenzoyl)-1,3-cyclohexanedione (NTBC) delays and in some cases fails to prevent disease progression to liver fibrosis, liver failure, and activation of tumorigenic pathways. Here we demonstrate for the first time a cure of HT1 by direct, in vivo administration of a therapeutic lentiviral vector targeting the expression of a human fumarylacetoacetate hydrolase $(F A H)$ transgene in the porcine model of HT1. This therapy was well tolerated and provided stable long-term expression of FAH in pigs with HT1. Genomic integration displayed a benign profile, with subsequent fibrosis and tumorigenicity gene expression patterns similar to wild-type animals as compared to NTBC-treated or diseased untreated animals. Indeed, the phenotypic and genomic data following in vivo lentiviral vector administration demonstrate comparative superiority over other therapies including ex vivo cell therapy and therefore support clinical application of this approach.
\end{abstract}




\section{Introduction}

Hereditary tyrosinemia type 1 (HT1) is an autosomal recessive inborn error of metabolism that is characterized by the inability to metabolize tyrosine. Caused by a deficiency of the fumarylacetoacetate hydrolase (FAH) enzyme, ${ }^{1} \mathrm{HT} 1$ causes an accumulation of toxic metabolites in the liver resulting in severe oxidative damage. These patients develop fibrosis, cirrhosis, high rates of hepatocellular carcinoma ( $\mathrm{HCC}$ ), and liver failure at a very young age if left untreated. ${ }^{2,3}$ Pharmacological treatment for HT1 exists in the form of 2-(2-nitro-4trifluoromethylbenzoyl)-1,3-cyclohexanedione (NTBC), a drug which inhibits the upstream enzyme 4-hydroxyphenylpyruvate dioxygenase (HPD) thereby reducing the formation of toxic metabolites responsible for liver disease progression, thus essentially converting the disease into hereditary tyrosinemia type 3 (HT3), with a more benign phenotype. ${ }^{4}$ For a majority of patients, NTBC greatly ameliorates the HT1 phenotype, and if initiated before one month of age seems to be able to reduce the development of HCC in at least the first three decades of life. ${ }^{5}$ However, it remains to be seen if the prevention of HCC persists during adult years, as introduction of this drug took place during the $1990 \mathrm{~s} .{ }^{6}$ A number of case reports and series point to the development of fibrosis, cirrhosis, and HCC despite NTBC therapy, especially when NTBC therapy was delayed. ${ }^{7-10}$ The frequency of HCC within the limited follow-up period available for patients treated with NTBC appears to be $<1 \%$ if started prior to 1 year of age, $7 \%$ if started between 1 and 2 years of age, $21 \%$ if started between 2 and 7 years of age, and $35 \%$ if started after 7 years of age. ${ }^{11}$ Therefore, these patients require lifelong HCC surveillance with alpha-fetoprotein (AFP) levels drawn every 3 to 6 months. ${ }^{6}$ In addition, HT1 patients treated with NTBC show progressive neurocognitive decline despite optimal NTBC dosing, possibly due to prolonged exposure to elevated tyrosine levels that occur on the drug. ${ }^{12-14}$ Although the exact 
pathophysiological mechanisms behind these deficits are not clear, ${ }^{6}$ two studies have identified a correlation between the phenylalanine to tyrosine ratio in these patients and IQ. ${ }^{15,16}$ This theory is further supported by the fact that HT3, the disease caused by a deficiency in the same enzyme that NTBC blocks, also presents with neurological manifestations including developmental delay. ${ }^{17-20}$ Complications attributed to NTBC treatment other than low IQ include attention deficits, memory and processing problems, psychomotor and behavioral issues, and impairment of executive function and social cognition. ${ }^{21-28}$ With daily dosing of NTBC, as well as frequent testing, many patients struggle with compliance issues related to NTBC therapy in the second and third decades of life. ${ }^{26,27}$ Non-compliance with NTBC therapy is a key factor in disease progression for HT1 patients. ${ }^{29}$ To date, the only therapeutic option for patients refractory to NTBC or with disease progression despite NTBC therapy, and the only curative treatment for HT1, is liver transplantation.

Gene therapy may offer HT1 patients an alternative, less invasive cure. Our group has previously demonstrated that ex vivo lentiviral (LV) gene transfer followed by autologous transplantation of corrected hepatocytes is curative in both mouse and pig models of HT $1 .{ }^{30}$ The ex vivo approach is effective, but autologous transplantation requires a partial hepatectomy in liver-based diseases to avoid the immune rejection that would occur with transplantation of allogeneic hepatocytes. Therefore, in vivo liver-directed gene therapy presents an attractive nonsurgical alternative for the treatment of inborn errors of metabolism of the liver.

Limited biochemical correction of the mouse model of HT1 has been achieved in vivo through retrovirus, adenovirus, and adeno-associated virus (AAV) mediated gene transfer, ${ }^{31-33}$ as well as through RNA-guided CRISPR/Cas9-mediated genome editing and metabolic pathway reprogramming. ${ }^{34,35}$ Thus far, none of these in vivo techniques have been tested in the more 
clinically relevant large animal model of HT1. The mouse model does not recapitulate some of the most critical aspects of the human phenotype as mice develop HCC independently of fibrosis and cirrhosis, while pigs and humans do not. ${ }^{36}$ In mice, HCC develops despite long-term NTBC treatment, and it does so in the setting of hepatocyte dysplasia, liver steatosis, anisocytosis, and lymphocytic infiltrates, but not widespread fibrosis and cirrhosis, as it does in human HT1 patients. ${ }^{37}$ It is well known at this point that mice develop HCC through an entirely different sent of molecular mechanisms when compared to pig and human, with differences including but not limited to: a basal metabolic rate in mice about seven times higher than in humans, affecting the levels of endogenous oxidants and other mutagens that are produced as by-products of normal oxidative metabolism; a difference in the way that many carcinogens are activated or neutralized; a proclivity towards the development of mesenchymal tissue cancers instead of the epithelial-cell layer carcinomas more common in humans; a high frequency of spontaneous cell immortalization in mice, possibly explained by their constitutive expression of telomerase, which is not usually expressed in human cells; a difference between the two species in the relative contributions of the p53 and retinoblastoma pathways to cell senescence; and key differences in the signaling requirements for cell transformation, where in mice perturbation of just the p53 and Raf-MAPK pathways seems to be sufficient to mediate tumorigenic conversion, while in human fibroblasts perturbation of six or more pathways is necessary to achieve the same outcome..$^{38,39}$ In addition, the efficiency of AAV-based therapy in humans is hampered by pre-existing neutralizing antibodies to the viral vector that develop after natural exposure to the wild-type virus and, in the case of liver disease, by immune-mediated clearance of AAV-transduced hepatocytes. ${ }^{40}$ The same is true of the immune response to the bacterial Cas9. ${ }^{41}$ AAV based systems have been used in adult patients with hemophilia and other diseases but these therapies 
would not be beneficial in HT1 patients as liver injury with subsequent hepatocyte death and regeneration leads to the loss of transgene expression in hepatocytes over time. ${ }^{42-44}$

In the human setting, HIV-derived LV vectors hold several potential benefits over AAV vectors. First, the low prevalence of HIV infection in humans decreases the chances of an immune response curtailing the therapy's effectiveness. ${ }^{45}$ Second, LV vectors are better suited for the treatment of certain diseases due to their ability to carry larger gene inserts. ${ }^{46}$ Finally, in HT1 patients and the pediatric population, where hepatocyte turnover is high, the use of integrating LV vectors may be key for stable, lifelong expression of the desired transgene, whereas episomal vectors will be progressively diluted out during cell replication until effectively lost. ${ }^{47}$ This advantage is amplified in HT1 as FAH-positive hepatocytes are selected for at the expense of injured FAH-negative hepatocytes.

Liver-based LV gene therapy has yielded promising results in a large animal model of hemophilia B; Cantore et al. showed that intraportal administration of a LV vector carrying the cFIX transgene is able to safely provide stable clinical benefit in dogs with this disease, and in doing so provided preclinical proof-of-concept for the in vivo use of LV vectors in liver gene therapy in their hemophilia model. ${ }^{48}$ In this study, we evaluated both the effectiveness and safety profile of in vivo liver-directed LV gene therapy for the treatment of a pro-cancerous metabolic disease, HT1. 


\section{Results}

In vivo portal vein delivery of a $\mathrm{LV}$ vector is well tolerated in pigs. To characterize the acute effects of LV vector administration in pigs and determine the optimal route of administration, we initially delivered a LV carrying the human FAH transgene expressed with the liver-specific alpha-1-antitrypsin (AAT) promoter (LV-FAH, Fig 1a) systemically via an ear vein. We found no significant differences in key cytokines, including TNF-alpha and IL-6, or white blood cell count response, between this animal and our reference lentiviral vector with green fluorescent protein when administered via portal vein (LV-GFP, Fig 1b, Suppl Fig 1a). In both cases, an acute increase in inflammatory markers was observed after administration, with peak concentrations being reached at approximately one hour after the end of injection, and a subsequent decrease to pre-treatment levels after 6-12 h. Circulating white blood cell counts decreased minimally following administration of the LV vector, and recovered to their predose range within hours. However, despite pre-treatment with an established immunosuppression protocol, ${ }^{48}$ systemic administration resulted in an anaphylactoid reaction with acute hypotension (mean pressure of $48 \mathrm{mmHg}$ ) that required vasopressor support (0.2 $\mathrm{mg}$ of epinephrine IV). To ensure this reaction was not related to the LV-FAH construct, we delivered LV-GFP systemically to another animal, and again observed a similar reaction to infusion of the vector (mean pressure $42 \mathrm{mmHg}$ ), requiring support for maintenance of vital functions (IV fluid bolus, $0.2 \mathrm{mg}$ of epinephrine IV and $1 \mathrm{mg}$ of atropine IV, data not shown).

Based on these results, we pursued a novel approach using percutaneous ultrasound-guided portal vein administration of the LV vector carrying the human FAH transgene in four $F A H^{-}$ /- pigs (Table 1). Animals that received portal vein delivery of the LV-FAH construct tolerated the infusion well with no acute complications and maintained stable vital signs throughout the 
procedure (Fig 1c, panels 1 and 2, Suppl Fig 1b). However, Pig No. 169 recovered from anesthesia and the infusion event but died acutely on day 2 post-treatment with no significant prodrome before death. Autopsy revealed no significant liver pathology or systemic inflammatory disease including pulmonary edema or microhemorrhage. However, this animal was found to have severe hypertrophic cardiomyopathy (Suppl Fig 2), which may have contributed to its sudden demise. Hypertrophic cardiomyopathy is a known complication in Landrace pigs with prevalence of up to $23 \%$ in some studies, and spontaneous sudden mortality of up to $18 \%$, particularly related to stress-inducing situations such as transportation. ${ }^{49,50}$ In the remaining animals, either early expression of the transgene ( 8 days) nor expansion of the corrected cells (45 days) had an effect on TNF-alpha levels (Fig 1c, panel 3), which remained within normal range.

\section{In vivo LV-FAH delivery cures the pig model of HT1 with no residual liver pathology.}

NTBC administration was discontinued at the time of vector infusion to stimulate expansion of newly transduced, FAH-positive hepatocytes. The three remaining animals were then cycled on and off NTBC based on clinical and weight parameters until they were able to maintain a healthy, NTBC-independent growth curve. Pig No. 166 achieved NTBC-independent growth at day 98 post treatment and Pig No. 167 at day 78 post-treatment, both after four cycles on the drug (Fig 2a). At the time of planned euthanasia (one year old, 337 days post-treatment), these pigs had been off NTBC for 239 and 259 days respectively with no health issues, and with and average weight gain of between 0.6 and $0.9 \mathrm{~kg}$ per day, which is normal for healthy, wild-type Large White and Landrace pigs. ${ }^{51,52}$

Biochemical parameters, including tyrosine levels and liver function tests, were measured to confirm correction of the disease phenotype. At 142 days post-treatment, tyrosine levels were 
$79 \mu \mathrm{M}$ in No. 166 and $63 \mu \mathrm{M}$ in No. 167. These levels are within normal limits for a wild-type pig and significantly lower than those of historical $F A H^{-/-}$controls $(\mathrm{p}=0.04$, Fig $2 \mathrm{~b}$, panel 1$)$. Normal tyrosine levels were maintained throughout all successive blood draws until the time of euthanasia (Suppl Fig 3). Aspartate amino transferase (AST), alkaline phosphatase (ALP), ammonia, and bilirubin levels were also normalized at day 142 post-treatment (Fig 2b, panels 25). All measured liver function tests were comparable to wild-type levels at this time point, despite animals having been off NTBC for 45-65 days. In fact, all liver function tests remained within normal limits throughout the experiment with the exception of ALP in both animals and gamma-glutamyl transferase (GGT) in one animal, which were elevated at only one time point (47 days post-treatment), between the third and fourth cycles on NTBC (Suppl Table 1). Additionally, AFP levels showed an age-appropriate decrease over time in both animals, remaining stable at $0.25 \mathrm{ng} / \mathrm{mL}$ at the end of the study (Fig 2c) which is identical to wild-type age matched controls. ${ }^{53}$

To allow for interim analysis, Pig No. 162 was euthanized at day 60 post-treatment after three cycles of NTBC. After harvesting the complete liver from No. 162, the presence of FAH-positive hepatocytes was evaluated. After examining over a hundred sections from the right, middle, and left lobes of the liver we found an even distribution of clonally expanded FAH-positive cells were present, constituting approximately $10 \%$ of the total liver cells (Fig 3b, top row panels 1 and 2). No active inflammation, fibrosis or necrosis was found in routine histology using H\&E or Masson's Trichrome staining (Fig 3b, top row panels 3 and 4). In contrast, no FAH-positive cells or liver abnormalities were found in Pig No. 169 48h post-treatment (Suppl Fig 4). The longerterm animals (Nos. 166 and 167) both underwent laparoscopic liver biopsies from the right and left lobes of the liver at 225 days post-treatment. Here, extensive liver repopulation with FAH- 
positive hepatocytes had occurred, totaling $69 \%$ and $78 \%$ of cells in the liver, respectively (Fig 3b, second and third row panels 1 and 2). As previously described after liver repopulation in $F A H^{-/-}$animals, individual hepatocyte clones were uniquely identified by variations in FAHstaining intensity between and within lobules. ${ }^{30}$ At this point, multiple abnormalities were seen in 50 to $80 \%$ of the liver of both animals, including inflammation and reversible fibrosis that could be compared to Stage 1-2 liver fibrosis on the METAVIR scoring system in humans, ${ }^{54}$ as well as hepatocyte inflammation and minimal bile stasis (Fig 3b, second and third row panels 3 and 4). These findings did not correlate with increased AFP staining. At 337 days post-treatment, samples were taken from the right, middle, and left lobes and after examining over 100 sections both animals showed near complete liver repopulation with FAH-positive hepatocytes (Fig 3a and $3 \mathrm{~b}$, fourth and fifth row panels 1 and 2) with near total resolution of the fibrosis and inflammation seen at day 225 along with restoration of normal hepatic architecture resembling wild-type liver (Suppl Fig 5). H\&E/Trichrome sections showed normal hepatocyte morphology and minimal fibrosis in $10-15 \%$ of the liver, which was confined to the periportal hepatocytes (Fig 3b, fourth and fifth row panels 3 and 4, Suppl Table 2). Importantly, adult wild-type animals show uniform hepatocellular morphology, with similar fibrosis limited to the connective tissue between lobules and periportal hepatocytes, and negligible AFP staining (Fig 3b, left column). Untreated or chronically undertreated $F_{A H} H^{-/}$adult pigs (No. 266, prior experiment, Fig 2a) develop cirrhosis, adenomas, and ultimately HCC at 12 months, as shown here with hepatocellular morphological derangement, extensive stage 4 fibrosis, and significant AFP staining (Fig 3c, right column). In the LV-FAH treated animals, no such lesions were present grossly after sectioning the entire organ into one centimeter slices, and none of these pathological features were present in any sections evaluated histologically. Untreated Pig No. 
266 showed appropriately elevated AFP at birth similar to Nos. 166 and 167 (Fig 2c) and to wild-type controls; however, at the time of necropsy (one year old) No. 266 showed increased AFP levels to $168 \mathrm{ng} / \mathrm{mL}$, further supporting the development of $\mathrm{HCC}$ in this animal as compared to Nos. 166 and 167, whose AFP levels at the time of necropsy are below $0.5 \mathrm{ng} / \mathrm{mL}$ (Fig 3d).

\section{Portal vein delivery of a $\mathrm{LV}$ vector is effective at targeting the liver with limited off-target}

bio-distribution. PCR screening of multiple tissue types was performed after portal and systemic LV-FAH delivery to evaluate for presence of the LV vector in target and non-target tissues. At 48 h post portal vein delivery (No. 169) positive vector presence was limited to all lobes of the liver (Fig 4a, top left). At sixty days (No. 162), evidence of vector integration was present only in the liver as well (Fig 4a, top right). Finally, at 337 days (Nos. 166 and 167) there was strong evidence of vector integration again in all lobes of the liver in both animals, with no evidence of off-target lentiviral integration elsewhere (Fig 4a, middle). In contrast, the pig in which LV-FAH was delivered systemically via ear vein injection showed no evidence of vector presence in the liver by standard PCR, whereas spleen, pancreas, duodenum, and lung were positive for LV-FAH at 14 days post-infusion (Fig 4a, bottom). At 60 days post-treatment, LV integration in the liver was measured at under one vector copy per genome (VCG), with this number increasing in long-term animals as FAH-positive hepatocytes expanded to repopulate the liver (Fig 4h).

\section{LV-FAH vector shows a benign integration profile in pig hepatocytes after in vivo delivery.}

In order to characterize the LV-FAH vector integration profile in hepatocytes after in vivo delivery, we performed next generation sequencing and bioinformatics analysis of hepatocytes at 
the time of euthanasia for animals at 60 (No. 162) and 337 days (Nos. 166 and 167, Fig 4, b-h). Mapping statistics are provided in Suppl Table 3.

Integration sites were present across the genome in all animals (Fig 4b). There were no significant differences in general LV integration profile in the liver between the two long-term animals; however, integration profile in the 60-day animal (No. 162) showed some unique characteristics. Interestingly, at 60 days, relative integration frequency was higher in exons as compared to introns, while at 337 days (Nos. 166 and 167) integration was more frequent in introns than in exons (Fig 4c), possibly indicating preferential propagation of cells with intronic integrations over the course of liver repopulation. Relative LV integration frequency remained similar across gene expression levels (Fig 4d). At both time points (all three animals), LV integration was extremely rare in $\mathrm{CpG}$-rich promoter regions relative to their percentage of the genome as compared to non-CpG islands $(\mathrm{p}=0.0001$, Fig $4 \mathrm{e})$, with a trend toward non-tumor coding genes for the longer-term animals (Fig 4f), possibly again indicating a selective preference for those hepatocytes over time. Lastly, integration was most prevalent downstream of transcription start sites, suggesting unlikely activation of any oncogenes from proximal integration of the AAT promoter-containing construct (Fig 4g). The top ten most common genes in terms of integration frequency for each animal and tissue type are shown in Table 2. LV-FAH integration was also found to be increased at the FAH and SERPINA1 loci suggesting a role for sequency homology in integration preference. Gene Set Enrichment Analysis (GSEA) demonstrated enrichment of the PI3K-Akt and TGFb signaling pathways only in No. 167, while No. 166 demonstrated no hits (Fig 4i). There were no changes in gene expression in any tumorrelated genes. 
Finally, RNAseq demonstrated expression profile similarities between wild-type, LV-FAH treated, and NTBC-treated pigs as compared to tumor-positive pig liver, particularly in cancerrelated and fibrosis-related genes (Fig 5a). Interestingly, while LV-FAH treated animals at 6 months showed an expression profile similar to both $F A H^{-/-}$sick or untreated animals (Fig 5b, top) and NTBC-sustained $F A H^{-/-}$animals (Fig 5b, bottom), by 12 months post-treatment where liver repopulation with healthy, FAH-positive hepatocytes had reached 100\%, the LV-FAH group is most similar to the wild-type expression profile. When gene expression was compared to 6 month and 12-month time points for our LV-FAH treated animals we found gene expression differences clustered into disease pathways of importance in the progression of tyrosinemia, including general tumorigenicity, inflammation and fibrosis and abdominal and digestive cancer (Fig 5c). 


\section{Discussion}

In this study, we demonstrated that lentiviral vector gene therapy is safe and effective for direct in vivo percutaneous portal vein administration in the relevant preclinical large animal model of HT1. Remarkably, the safety findings in this experiment support the in vivo application of this integrating vector with the absence of promotor- or transgene-specific adverse events and in the setting of complete resolution of the biochemical and histologic features of tyrosinemia that lead to progressive systemic and liver disease. Experimental $F A H^{-/-}$pigs were cycled on and off NTBC for selection of FAH-positive hepatocytes and were cured of their disease after a single intraportal dose of the LV vector carrying the human $F A H$ under control of the liver-specific HCR-AAT promoter.

Percutaneous portal vein infusion of LV-FAH was well tolerated, with no systemic inflammatory reaction. Vitals were closely monitored for $48 \mathrm{~h}$ after administration of the vector and showed a minimal changes in heart rate and blood pressure with stable temperatures. When the same vector was delivered systemically, however, animals developed an acute hypotensive anaphylactoid-like reaction requiring vasopressor support, likely related to a transient rise in circulating cytokines TNF- $\alpha$, IL-6, IL-8 and INF after exposure to LV particles, ${ }^{55}$ but returning to baseline by 6-12 h post-injection. Naldini and colleagues have previously described similar results after an open surgical approach with portal vein administration of LV carrying a factor IX transgene in dogs. ${ }^{48}$ This inflammatory response has been seen previously in mice exposed to LV particles, thought to be related to activation of hepatosplenic plasmacytoid dendritic cells triggered by contaminating nucleic acids or by the presence of the LV particles themselves. ${ }^{55}$ These results could be avoided or reduced by optimization of pre-existing immunosuppression protocols, ${ }^{48}$ by species-specific dose titering to minimize systemic exposure, and through the use 
of the percutaneous delivery methods described in this study. Percutaneous portal vein cannulation is an advanced but obtainable skill that would allow for liver-directed gene therapy in humans to be administered under conscious sedation as opposed to general anesthesia, therefore avoiding the confounder and added physiologic stress of undergoing an open operation resulting in activation of significant inflammatory pathways seen with surgery.

Percutaneous portal vein delivery was also demonstrated to be very effective at targeting the liver in contrast to systemic delivery, limiting biodistribution to off-target tissues. Extensive PCR analysis showed highly restricted extrahepatic tissue targeting when compared to systemic administration. Although examination of biodistribution after systemic delivery was only performed in one animal at 14 days post-treatment, biodistribution results in the portal vein delivery group were consistent between all four animals at all timepoints, including early (48 h), mid (60 days), and late (337 days). Initial lentiviral integration into the liver was measured at less than one VCG, well below FDA recommendations of five VCG. ${ }^{56}$ This number rapidly increased to ten in our long-term animals as corrected hepatocytes expanded to repopulate the liver, possibly suggesting natural selection of hepatocytes with higher VCG or more robust enzymatic activity.

Importantly, liver-directed in vivo LV gene therapy cured HT1 in our experimental animals and did so in a more rapid timeframe than ex vivo gene therapy using the same vector construct, therefore offering less opportunity for liver damage. Two long-term pigs demonstrated NTBC independence at day 78 to 98 post-treatment, after only four cycles on the drug. By comparison, our previous ex vivo study resulted in NTBC independence at 93 to 150 days post-treatment, after four to six cycles on the drug. ${ }^{30,57}$ In addition, all liver function tests in experimental 
animals were found to be at wild-type levels at four and five months post-treatment, with AFP levels showing an age-appropriate decrease that was maintained throughout the study.

By the end of the study (337 days), liver histology in our experimentally treated animals mirrored the normal hepatic architecture found in our wild type age-matched controls. We found no evidence of chronic liver damage or increased AFP staining in our experimental animals even after eight months off the protective drug NTBC. This is especially important because we have also demonstrated here that $\mathrm{FAH}^{-/}$animals that do not receive gene therapy and are chronically undertreated with NTBC develop extensive fibrosis within weeks and can progress to hepatic adenomas within 6 months of life and HCC prior to a year of life. Importantly, HT1 pigs develop HCC in a fibrosis-dependent manner like humans and unlike mice, ${ }^{37,58,59}$ which makes the FAHdeficient pig an ideal preclinical model to approximate the potential deleterious effects of lentiviral therapy and NTBC treatment/withdrawal in humans. Previous studies using a nonlentiviral retrovirus (GIFSvNa) in mice to treat HT1 in vivo showed metabolic correction of the phenotype but increased rates of HCC. At that time the assumption was made that the increased HCC rates were due to a number of uncorrected hepatocytes that persisted and then progressed to HCC. This safety concern has precluded gene therapy development for HT1 for more than two decades, while our recent data in the more clinically relevant pig model show that this concern is outdated. The reasoning for this comes from our updated understanding of how HCC develops in mice, pigs, and humans.

Mice develop adenomas and HCC through a different set of molecular derangements when compared to humans and pigs; in fact, the pig model of HCC has been shown to develop HCC through a similar set of molecular derangements as seen in most human HCCs, including increased TP53 and KRAS expression, sustained angiogenesis resulting from overexpression of 
PDGFA and ANGPT2, evasion of apoptosis through overexpression of TWIST1 and other mechanisms, reactivation of TERT for telomere maintenance, and Wnt signaling activation. ${ }^{60,61}$ In humans, including HT1 patients, HCC develops in the setting of an extensively inflamed, fibrotic, or cirrhotic liver with the exception of the fibrolamellar variant or rare cases of HBVrelated oncogenicity, ${ }^{62}$ while in $F A H^{-/-}$mice $\mathrm{HCC}$ has been found to appear in the setting of no such alterations. ${ }^{32}$ This is consistent with the observation that the tissue setting in which HCC develops is markedly different in humans and rodents, where chronic hepatitis and cirrhosis are uncommon lesions in the livers of rats and mice that develop HCC. ${ }^{36}$ Therefore, mice develop adenomas and HCC in the liver through an inflammatory and fibrotic-independent process, where in humans and pigs, especially in the setting of HT1, adenomas and HCC develop almost exclusively in the backdrop of chronic fibrosis and cirrhosis, making the absence of these findings in the liver protective. We show histologically that by the end of therapy there is no evidence of any chronic inflammatory changes anywhere in the liver and this finding is supported by the RNA sequencing results discussed later. We also did not find any evidence of adenomas or HCC in either of our long-term experimental animals, consistent with our results with ex vivo therapy, ${ }^{30}$ nor did we find enrichment of any tumor-related pathways on GSEA. In addition, these animals were cycled on and completely off $\mathrm{NTBC}$; this regimen could in theory be altered to involve chronic low doses of the drug, for example, in order to reduce the risk of fibrosis as FAH-positive hepatocytes expand to repopulate the liver, as well as avoid the porphyria-like crises and coagulopathy that untreated FAH deficiency causes in HT1 patients. ${ }^{63}$

Further advocating for the safety of this approach, the LV-FAH vector showed a benign integration profile in pig hepatocytes after in vivo delivery. Evaluation of 17,000-39,000 unique integration sites in all animals revealed no genotoxic events and neither favored integration in 
tumor-coding regions of the genome or in $\mathrm{CpG}$-rich promoter regions, and this correlation remained uniform for all liver tissue analyzed (Suppl Table 3). This is consistent with previous studies, where lentivirus tends to integrate into gene coding regions without an obvious preference for transcription start sites, promoter regions or other regulatory sites of the genome. ${ }^{64,65}$ These data, taken together with our previous work showing no evidence of genotoxicity of in vivo LV-FAH in an HCC-prone mouse model ${ }^{66}$ and GSEA showing only enrichment of the PI3K-Akt and TGFb signaling pathways in one animal, establish a favorable safety profile, particularly since hepatocytes have been shown to be susceptible to insertional mutagenesis. ${ }^{67,68}$ In our study, we observed integration bias in liver DNA beyond the wellestablished expectations reported previously. ${ }^{69}$ We found that in addition to these previously reported biases, LV-FAH integration was increased at the FAH and SERPINA1 loci, which may represent an integration bias based on sequence homology.

Gene expression associated with inflammation, fibrosis, and $\mathrm{HCC}$ in the liver has been well characterized..$^{70}$ RNAseq analysis at one year of age from undertreated NTBC animals that had developed HCC was compared to age-matched controls of wild-type, adequately NTBCmaintained, and LV-FAH treated pigs. Relative to each other, there were significant patterns involving inflammation, fibrosis, and cancer-related genes that suggest LV-FAH treatment restores a wild-type phenotype by 12 months post-treatment. Remarkably, animals treated adequately with NTBC showed increases in these liver injury and tumor-associated genes, which may explain the robust data now available in humans demonstrating development of cirrhosis and HCC in HT1 patients despite optimal and compliant NTBC therapy. ${ }^{7-10,71,72}$ In addition, like wild-type animals, pigs treated with LV-FAH have normal, undetectable levels of AFP at one year, which is in stark contrast to undertreated NTBC FAH-deficient animals. This further 
supports our proposal of LV-FAH as an acceptable advancement in the treatment of HT1 in lieu of chronic NTBC maintenance therapy, which does not prevent development of inflammation, fibrosis, and HCC due to residual progression of tyrosine metabolism through the degradation pathway resulting in production of toxic metabolites. Furthermore, FAH-deficient patients optimally treated with NTBC are relegated at best to suffer from the HT3 phenotype, which can be debilitating over time. ${ }^{21-23,26}$ HT1 patients are still in need of a true cure, such as that offered by LV-FAH.

Finally, the most compelling aspect of this model and disease is the characteristic expansion of FAH-positive hepatocytes. Genetically modified hepatocytes in the HT1 pig model provide an exquisitely sensitive tool to assess gene therapy platforms, including LV, for mutagenesis with subsequent robust clonal expansion of any deleterious clones. We provide evidence here that the HT1 pig model can develop HCC in the absence of gene therapy and adequate NTBC dosing, establishing the significance of the absence of tumorigenic integration events or activation of oncogenic pathways in LV-FAH treated animals and providing convincing safety data for consideration of human application. This work contributes to a growing body of evidence that LV vectors have a lower genotoxic risk when compared to gamma-retroviral vectors. ${ }^{73-75}$ Although multiple strategies have and are being employed to increase the safety of lentiviral vectors in general, ${ }^{76,77}$ patients participating in clinical trials involving lentiviral vectors will continue to require close long-term surveillance. It is worth noting that HT1 patients already require frequent cancer-related surveillance and $\mathrm{HCC}$ screening, ${ }^{5}$ and this requirement would not be fundamentally altered after curative gene therapy to treat HT1.

There are a number of limitations to our study. The main and most significant limitation is the small number of animals treated and analyzed. As is often the case with preclinical large animal 
studies, inclusion of a large sample size would be cost-prohibitive. Results must be interpreted in the context of limited data obtained from a small number of animals followed for a relatively short period of time. However, the importance of testing new therapies in preclinical models that, unlike rodent models, fully recapitulate the human disease phenotype must not be undervalued, compounded with the fact that this particular model has demonstrated the ability to develop HCC within the relatively short timeframe studied. Doses were based on previous liver-directed in vivo LV large animal studies, ${ }^{48}$ as well as our own results in mice. Further dose-response studies are warranted to investigate the effects of dose on biodistribution and to find a balance between rapid cure with limited NTBC cycling and mitigation of systemic exposure leading to extrahepatic integration events. The doses used in this study would require large-scale manufacturing in adult or pediatric human patients, but new technology such as hollow fiber reactors and suspension culture processes are very rapidly decreasing manufacturing hurdles. ${ }^{78,79}$ Strategies to decrease the LV dose needed could also include temporary Kupffer cell knockdown prior to LV administration in order to decrease vector clearance in the liver. ${ }^{80}$ These further studies will be crucial to establish optimal in vivo lentiviral treatment protocols for clinical use.

In conclusion, our study provides further evidence that in vivo lentiviral liver-targeted gene therapy via direct percutaneous portal vein delivery is safe and effective. These data support the consideration of in vivo administration of lentiviral vectors for the treatment of HT1 and other disorders that could be cured with stable integrating gene delivery. 


\section{Online Methods}

Vector production. We have designed a LV vector containing the human $F A H$ gene under control of a liver-specific promoter (HCR-AAT; hepatic control region enhancer and alpha-1 antitrypsin promoter), currently being trialed in humans for the treatment of hemophilia B. ${ }^{40} \mathrm{~A}$ schematic representation of this vector is provided in Fig 1A.

In order to generate viral vectors, the LV-SFFV-eGFP or LV-AAT-huFAH expression construct, together with the packaging plasmid p8.91 and the vesicular stomatitis virus glycoprotein Gencoding plasmid pVSV-G, was transfected into $293 \mathrm{~T}$ cells using $1 \mathrm{mg} / \mathrm{ml}$ polyethylenimine (Polysciences, Warrington, PA). Viral supernatant was harvested 48 and $72 \mathrm{~h}$ after transfection, filtered through a $0.45-\mu \mathrm{m}$ filter, and concentrated by ultracentrifugation $(25,000 \mathrm{rpm}, 1.5 \mathrm{~h}$ at $4^{\circ} \mathrm{C}$ ). After resuspension in serum-free media (DMEM, Thermo Fisher Scientific, Waltham, MA), LV vectors were aliquoted and stored at $-80^{\circ} \mathrm{C}$. Vector titers were determined by p24 enzyme-linked immunosorbent assay and qPCR using the Lenti-X Provirus Quantitation Kit (Clontech, Mountain View, CA).

Animals and animal care. For pig gene therapy experiments, male and female $F A H^{-/-}$pigs were used. $\mathrm{FAH}^{-/-}$pigs were produced in a 50\% Large White and 50\% Landrace pig. To obtain a homozygous age-matched cohort, heterozygous females were bred with homozygous males, yielding an expected prevalence of homozygosity in the litter of 50\%. After birth, a notch of ear tissue from each piglet was obtained for genotypic confirmation through PCR as previously described. ${ }^{81,82}$ Piglets determined to be homozygous were reserved for experimental purposes.

NTBC was obtained courtesy of Dr. Vadim Gurvich (University of Minnesota, Minneapolis, $\mathrm{MN}$ ) and was administered mixed in food at a dose of $1 \mathrm{mg} / \mathrm{kg} /$ day with a maximum of 25 
mg/day. All animals remained on NTBC until the time of LV-FAH treatment, after which NTBC administration was discontinued. Pigs were monitored daily for weight loss, and NTBC was reinitiated for seven days if weight loss of $15 \%$ or more of body weight occurred or other signs of morbidity were present. Animals were cycled on and off NTBC in this fashion to stimulate expansion of corrected FAH-positive cells. Phenotype correction was assumed when an animal was able to thrive without NTBC treatment. For pig biodistribution experiments, male and female wild-type domestic pigs were used (Manthei Hog Farm, Elk River, MN).

Pig experiments. Pigs were pre-treated according to a previously established immunosuppression protocol. ${ }^{48}$ Ear vein injection of LV-FAH was performed in one six-weekold $F A H^{-/-}$pig, at a dose of approximately $2 \times 10^{10}$ transducing units (TU)/kg. In this case, the vector solution was infused through a 24-gauge intravenous catheter. Portal vein injection of LVGFP at the same dose was performed in one heterozygous $F A H^{+/-}$pig, also at six weeks of age.

Portal vein injections of LV-FAH at a dose of approximately $2 \times 10^{10}$ transducing units (TU)/kg were then performed in four $\mathrm{FAH}^{-/-}$pigs at six weeks of age. The portal vein was identified using a 2 to $5 \mathrm{MegaHz}$ transducer (Fujifilm SonoSite, Inc., Bothell, WA), and an 18-gauge 5-in needle was directed percutaneously towards the main portal vein prior to its bifurcation for manual infusion of the vector solution.

Two reference pigs were evaluated at one year of age (Nos. 266 and 158) to demonstrate the development of HCC in this $F A H^{-/-}$pig model. No. 158 was cycled on and off an appropriate dose of NTBC, while No. 266 was chronically underdosed to allow progression of the disease without mortality (Fig 2a). 
Biochemical analysis. Complete blood counts were determined by analysis with the VetScan HM5 analyzer (Abaxis, Union City, CA) according to the manufacturer's instructions. Liver function tests and alpha-fetoprotein (AFP) levels were determined in serum, and ammonia levels were determined in plasma using standard protocols by the Mayo Clinic's central clinical laboratory. Tyrosine values in plasma were determined using liquid chromatography and tandem mass spectrometry by the Mayo Clinic's biochemical genetics laboratory. TNF-alpha, IL-6, and IL-8 levels were determined in serum through enzyme-linked immunosorbent assay by the Mayo Clinic's immunochemical laboratory. Liver function tests and tyrosine levels were compared to historical untreated $F A H^{-/-}$and wild-type controls.

Histopathological analysis. At the time of liver biopsy, two liver samples were collected, one from the left and one from the right lobes. After euthanasia, liver samples were collected from sixteen different areas spanning the entire organ. These areas were labeled as follows: left lateral lobe anterior superior, left lateral lobe anterior inferior, left lateral lobe posterior superior, left lateral lobe posterior inferior, left medial lobe anterior superior, left medial lobe anterior inferior, left medial lobe posterior superior, left medial lobe posterior inferior, right medial lobe anterior superior, right medial lobe anterior inferior, right medial lobe posterior superior, right medial lobe posterior inferior, right lateral lobe anterior superior, right lateral lobe anterior inferior, right lateral lobe posterior superior, and right lateral lobe posterior inferior. For histological analysis, tissue samples were fixed in 10\% neutral buffered formalin (Azer Scientific, Morgantown, PA) and processed for paraffin embedding and sectioning. H\&E and Masson's Trichrome staining were prepared by means of standard protocols. H\&E and Trichrome slides were evaluated by a pathologist for presence of liver injury and fibrosis. FAH immunohistochemistry using a polyclonal rabbit anti-FAH primary antibody ${ }^{83}$ was performed with a Bond III automatic stainer 
with a 20-minute antigen retrieval step using Bond Epitope Retrieval Solution 2 (Leica, Buffalo Grove, IL), and stained with diaminobenzidine (Leica, Buffalo Grove, IL). GFP immunohistochemistry using a monoclonal rabbit anti-GFP primary antibody (Cell Signaling, Danvers, MA) was performed using the same process. H\&E and Trichrome slides from each of the sixteen liver areas sampled were reviewed by an independent blinded liver pathologist that estimated the percent of fibrotic liver tissue in each slide examined. Quantification of FAHpositive cells was performed using cytoplasmic stain algorithms in Aperio ImageScope. Entire slides from each lobe of the liver were analyzed and quantified for each animal. Reported results are the total percentage of cytoplasmic FAH positivity among the cells in each slide.

Vector integration analysis. Genomic DNA was isolated from snap-frozen tissue fragments using a Gentra Puregene Tissue Kit (Qiagen, Hilden, Germany). Representative samples were taken from sixteen distinct areas of the liver and pooled for analysis. Three pairs of primers were designed to amplify integrated vectors in the pig genome, targeting the 3' LTR and 5' LTR regions as well as the transgene of interest. For LV-GFP, these primers were: 3' LTR (5'CTGTTGGGCACTGACAATTC-3' and 5'-TAACTAGAGATCCCTCAGACCC-3'), 5' LTR (5'-GGATGTCTTCAATCAGCCTA-3' and 5'-GGCTTAGAGTCATCAGGTTT-3'), and GFP (5'-CCTGAAGTTCATCTGCACCA-3' and 5'-GACAACCACTACCTGAGCAC-3'). For LVFAH, these primers were: 3' LTR (5'-TGTGACTCTGGTAACTAGAGATCCCTC-3' and 5'TTGCCTTGGTGGGTGCTACTCCTAATG-3'), 5' LTR (5'-GGATGTCTTCAATCAGCCTA3' and 5'-GGCTTAGAGTCATCAGGTTT-3'), and FAH (5'-TGTTGGAACTGTCGTGGAAG3' and 5'-AGCAGTGGGTTCCCTAGTTA-3'). PCR was performed with GoTaq DNA Polymerase (Promega, Madison, WI) using an initial denaturation step at $98^{\circ} \mathrm{C}$ for $3 \mathrm{~min}$, followed by 30 cycles at $98^{\circ} \mathrm{C}$ for $30 \mathrm{~s}, 58^{\circ} \mathrm{C}$ for $30 \mathrm{~s}$, and $72^{\circ} \mathrm{C}$ for $40 \mathrm{~s}$, and finally at $72^{\circ} \mathrm{C}$ for 
5 min. PCR products were visualized by means of $2 \%$ TBE agarose gel electrophoresis with ethidium bromide staining.

\section{Amplification, next-generation sequencing (NGS), and genomic DNA mapping of lentiviral}

integration sites. Genomic DNA was isolated from snap-frozen tissue fragments using a Gentra Puregene Tissue Kit (Qiagen, Hilden, Germany). Ligation-mediated PCR (LM-PCR) was used for efficient isolation of integration sites. Restriction enzyme digestions with MseI were performed on genomic DNA samples; the digested DNA samples were then ligated to linkers and treated with ApoI to limit amplification of the internal vector fragment downstream of the $5^{\prime}$ LTR. Samples were amplified by nested PCR and sequenced using the Illumina HiSeq 2500 Next-Generation Sequencing System (San Diego, CA). After PCR amplification, amplified DNA fragments included a viral, a pig and sometimes a linker segment. The presence of the viral segment was used to identify reads that report a viral mediated integration event in the genome. The reads sequenced from these DNA fragments were processed through quality control, trimming, alignment, integration analysis, and annotation steps.

Quality control of the sequenced read pairs was performed using the FASTQC software. The average base quality of the sequenced reads was $>$ Q30 on the Phred scale. Uniform distribution of $\mathrm{A}, \mathrm{G}, \mathrm{T}$ and $\mathrm{C}$ nucleotides was seen across the length of the reads without bias for any specific bases. The number of unknown "N" bases was less than $1 \%$ across the length of the reads. High sequence duplication ( $>80 \%$ ) was observed, however, this was anticipated due to the nature of the experiment and the amplification of specific library fragments.

We used Picard software's (http://broadinstitute.github.io/picard/) insert size metrics function to calculate the average fragment length per sample. This metric averaged across all the samples 
was 158 base pairs (bps) long with an average standard deviation of 29bps. Since $150 \mathrm{bp}$ long reads were sequenced, most of the paired read one (R1) and read two (R2) reads overlapped, providing redundant information. R2 reads were therefore not considered in the analysis.

Reads were trimmed to remove the viral sequence in two separate steps. In step 1, the viral sequence was trimmed from the $\mathrm{R} 1$ reads using cutadapt ${ }^{84}$ with a mismatch rate $(\mathrm{e}=0.3)$ from the 5 ' end of each read. In step 2, if the linker sequence was present, it was similarly trimmed from the 3 'end of the read. Trimmed reads with a length less than $15 \mathrm{bps}$ were removed from the rest of the analysis to reduce ambiguous alignments. Untrimmed reads were also removed from the rest of the analysis because they did not contain a viral segment that could be used as evidence of a viral mediated integration.

The remaining R1 reads were aligned to the susScr11 build of the pig reference genome using BWA-MEM in single-end mode. ${ }^{85}$ Default BWA-MEM parameters were used. The reads used to identify genomic points of viral integrations had to be uniquely mapped to the genome with a BWA-MEM mapping quality score greater than zero.

An integration point was defined by the position of R1's first aligned base on the susScr1 genome. Unique integration points were identified across the genome without any constraints on coverage. However, for downstream annotation and analysis, only those integration points with 5 or more supporting reads were used to minimize calling false positive integration points.

Locations of integration points were categorized into: exons, introns, 3 prime UTRs, 5 prime UTRs and intergenic regions using information extracted from the susScr11 refflat file maintained by UCSC's Genomics Institute. To avoid conflict of feature categorization arising from multiple overlapping gene definitions, only the definition of the longest gene was used to 
annotate integration points. Additional features where computed including: the distance to the nearest transcription start site (TSS), makeup of CpG-rich regions of the genome ("CpG islands"), and enrichment to tumor-associated genes. This tumor gene list was comprised of 745 clinically validated tumor-associated human genes (from Mayo Clinic internal data). Those genes were related to their pig homologs based on GenBank's gene names.

In order to look for selective enrichment of any specific biological pathways, the genes identified as integration sites were entered into the WEB-based GEne SeT AnaLysis Toolkit. ${ }^{86}$ Results were identified using the over-representation analysis (ORA) method with identified KEGG pathways. A false discovery rate (FDR) of $<0.05$ was considered significant.

RNA sequencing. The mRNA-Seq data were aligned with STAR version $2.5 .2 b^{87}$ to the Sscrofa11.1 assembly of the pig genome. Raw gene expression was quantified with the featureCounts software. ${ }^{88}$ Normalization and differential expression analyses were performed using $\mathrm{R}$ version 3.5.3 with the edgeR version $3.24 .3^{89}$ and limma version 3.38.3 packages. Network and functional term enrichment analyses were processed through Qiagen's Ingenuity application ${ }^{90}$ on the identified differentially expressed genes. Heat maps visualizing the z-scores of the relevant genes' normalized expression values were created using the clustvis web tool. ${ }^{91}$

Statistics. Numerical data are expressed as mean ( \pm standard deviation). Biochemical comparisons between groups were performed using the Mann-Whitney U test. $\mathrm{P}<0.05$ was considered statistically significant. Statistical analysis was performed using GraphPad Prism software version 7.03 . 
bioRxiv preprint doi: https://doi.org/10.1101/2021.01.02.425079; this version posted March 16, 2021. The copyright holder for this preprint (which was not certified by peer review) is the author/funder. All rights reserved. No reuse allowed without permission.

Study approval. All animal procedures were reviewed and approved by Mayo Clinic's Institutional Animal Care and Use Committee, and all animals received humane care for the duration of the study. 


\section{Author Contributions}

C.T.N. designed the study, conducted experiments, performed animal care, acquired and analyzed data, and wrote the manuscript. C.J.VL. conducted experiments and acquired and analyzed data. K.L.A. conducted experiments, performed animal care, and acquired data. R.D.H. designed the study and analyzed data. Z.D. and R.M.G. conducted experiments and acquired data. L.G.H. performed data collection and animal curation. G.M., A.B., D.O., and J.P.K. analyzed data. R.A.K. and J.B.L. designed the study, conducted experiments, analyzed data, and wrote the manuscript. 


\section{Acknowledgements}

Funding for this study came from Children's Hospitals of Minnesota and additional support was provided by Regenerative Medicine Minnesota. We thank L. Gross and L. Acosta for histology support; S. Krage and J Pederson for surgical support; G. Mondal for assistance with bioinformatics data. 
Table 1. Gene therapy dose in treated pigs. Four animals (two male and two female) were assigned to dosing as indicated below. Animals were surgically anesthetized and dosed with $2 \times 10^{10} \mathrm{TU} / \mathrm{kg} \mathrm{LV}-\mathrm{FAH}$ directly via the portal vein.

\begin{tabular}{|c|c|c|c|c|c|c|c|c|c|}
\hline Pig & Genotype & Sex & $\begin{array}{c}\text { Age } \\
\text { (weeks) }\end{array}$ & $\begin{array}{c}\text { Weight } \\
\text { (kg) }\end{array}$ & Vector & Route & $\begin{array}{l}\text { Dose } \\
(\mathrm{mL})\end{array}$ & $\begin{array}{c}\text { Dose } \\
\text { (TU/kg) }\end{array}$ & $\begin{array}{c}\text { Study } \\
\text { Duration }\end{array}$ \\
\hline No. 169 (Carmen) & $F A H^{-/-}$ & $\mathrm{F}$ & 6 & 11.8 & LV-FAH & Portal & 150 & $2.00 \times 10^{10}$ & 2 days ${ }^{*}$ \\
\hline No. 162 (Santiago) & $F A H^{-/-}$ & $\mathrm{M}$ & 6 & 10.4 & LV-FAH & Portal & 135 & $2.04 \times 10^{10}$ & 60 days \\
\hline No. 166 (Pepe) & $F A H^{-/-}$ & $\mathrm{M}$ & 6 & 10.8 & LV-FAH & Portal & 140 & $2.04 \times 10^{10}$ & 337 days \\
\hline No. 167 (Lola) & $F A H^{-/-}$ & $\mathrm{F}$ & 6 & 13.6 & LV-FAH & Portal & 170 & $1.96 \times 10^{10}$ & 337 days \\
\hline No. 762 & $F A H^{-/-}$ & $\mathrm{M}$ & 6 & 10.2 & LV-FAH & Systemic & 224 & $2.00 \times 10^{10}$ & 14 days \\
\hline No. 764 & $F A H^{+/-}$ & $\mathrm{M}$ & 6 & 9.2 & LV-GFP & Portal & 305 & $2.00 \times 10^{10}$ & 13 days \\
\hline
\end{tabular}

* Animal recovered from anesthesia slowly and was found dead on Day 2 due to a suspected inflammatory reaction; TU - transducing units 
a

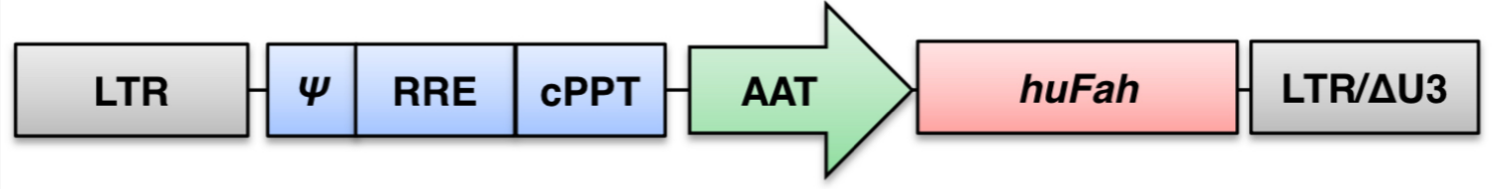

b

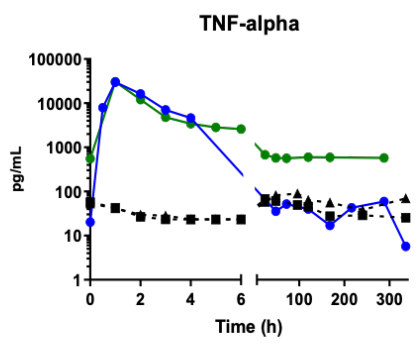

C

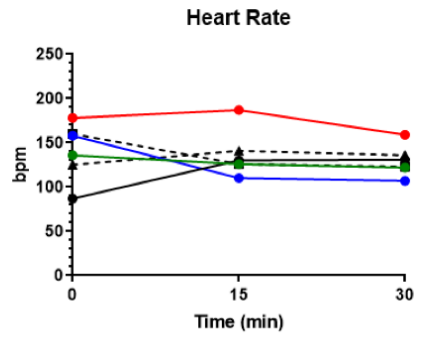

IL-6

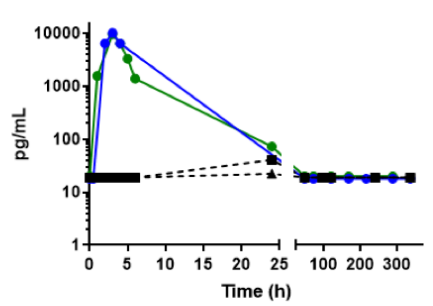

Mean Arterial Pressure

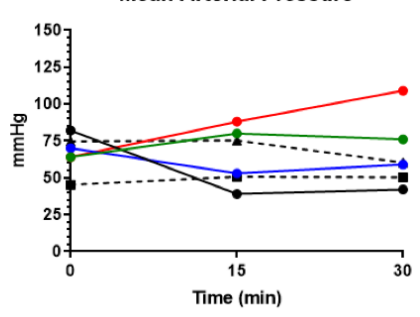

White Blood Cells

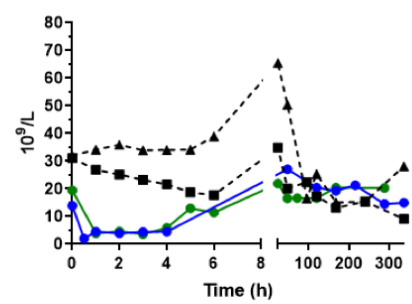

$\rightarrow$ LV-FAH Sytemic

- LV-GFP Portal

-. Portal Control

- Systemic Control

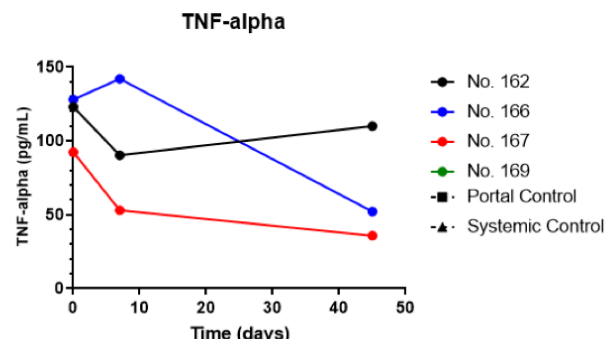

Figure 1. LV-FAH administration to pigs. (a) Schematic representation of the lentiviral vector carrying the human $F A H$ cDNA under control of the human alpha-1-antitrypsin (AAT) promoter. LTR, long terminal repeat; $\Psi$, psi packaging sequence; RRE, Rev-responsive element; cPPT, central polypurine tract; LTR/ $\Delta \mathrm{U} 3,3^{\prime}$ long terminal repeat with deletion in $\mathrm{U} 3$ region. (b) Acute inflammatory response after systemic LV-FAH administration compared to reference portal vein LV-GFP delivery. No significant differences in TNF-alpha or IL-6 concentrations or white blood cell count were found between the two routes of administration. Note that $30,000 \mathrm{pg} / \mathrm{mL}$ represents the upper limit of detection for the TNF-alpha assay. (c) Vital signs and TNF-alpha levels during and after portal vein delivery of LV-FAH in four $F A H^{-/-}$animals. Heart rate and blood pressure remained stable throughout infusion, and TNF-alpha levels were within normal range immediately post-infusion and at 8 and 45 days post-treatment. 
a
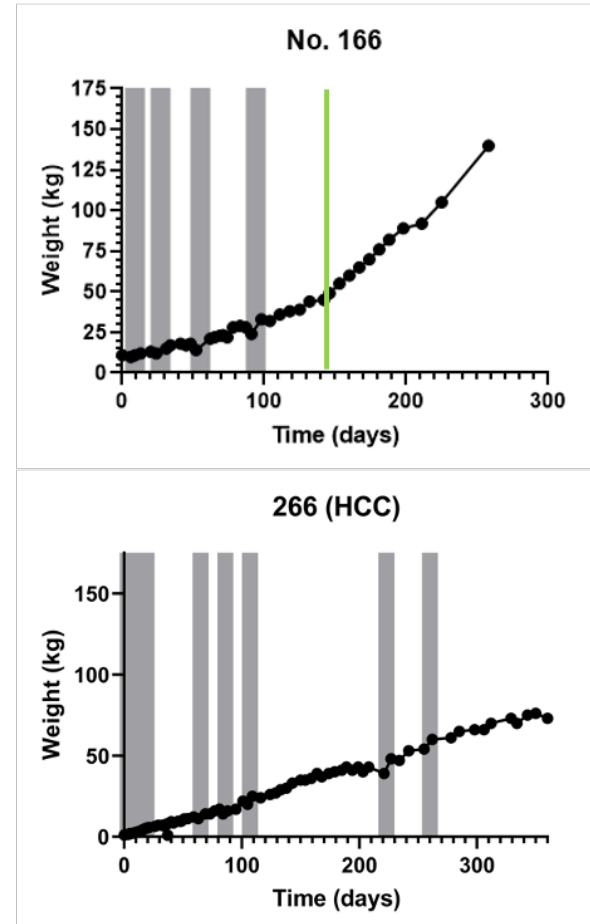

No. 167

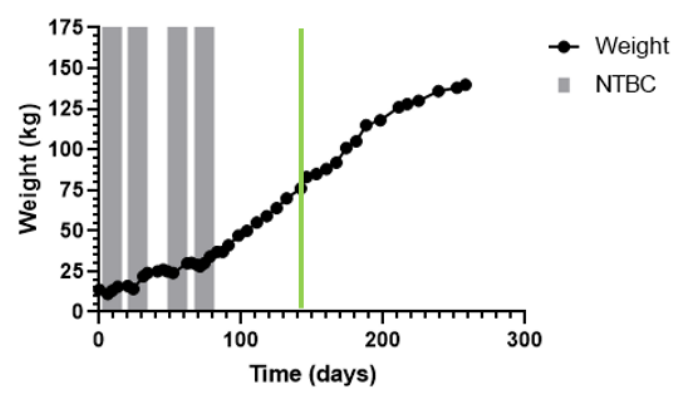

158 (Control)

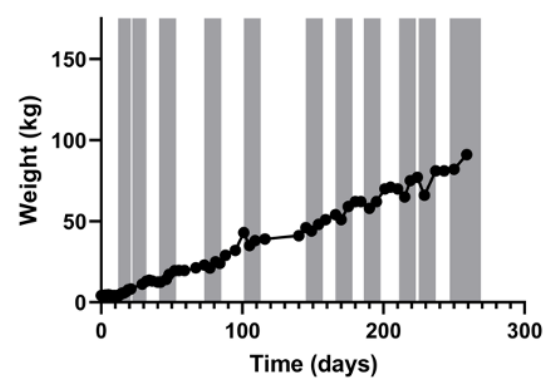

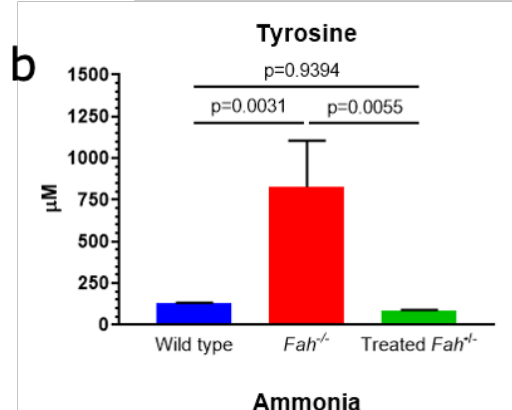
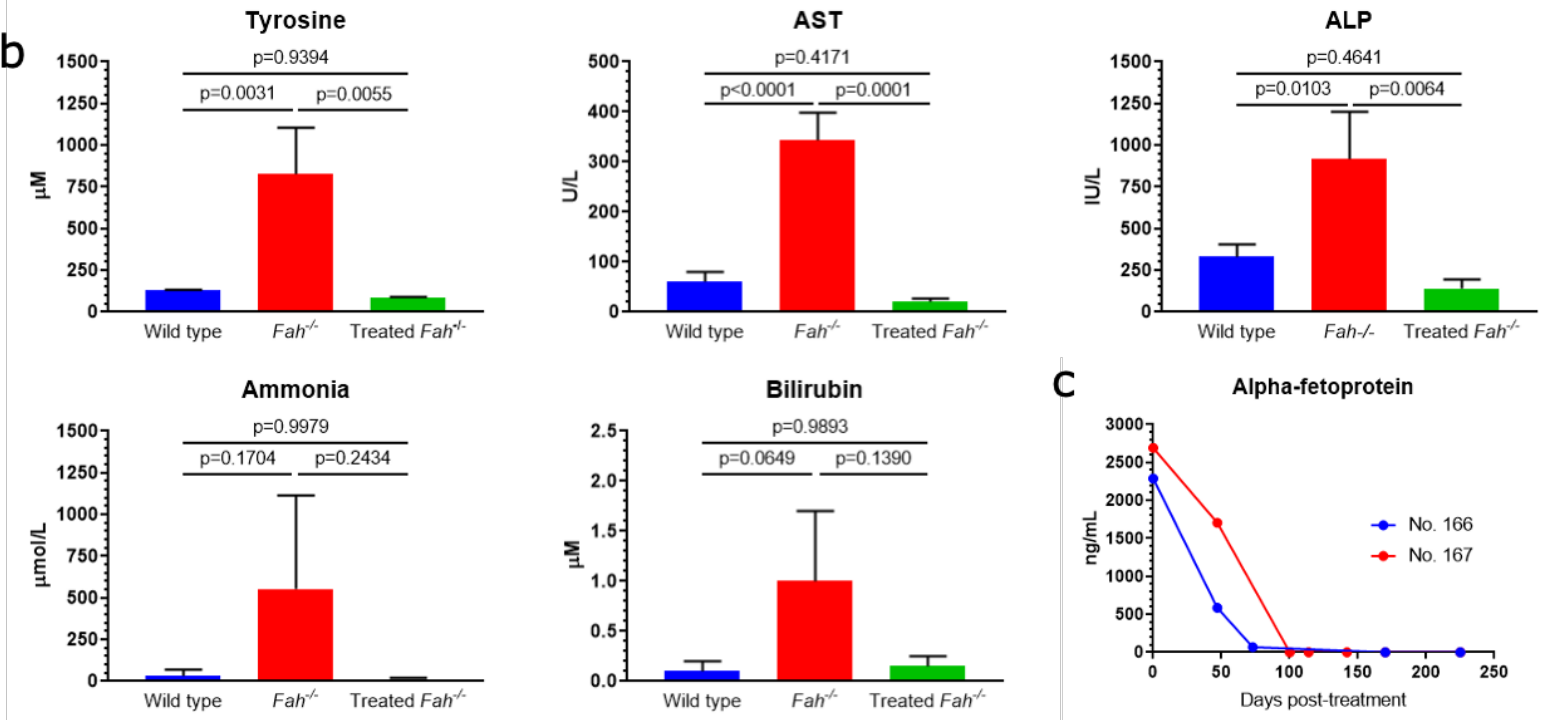

Figure 2. NTBC-independent weight gain and correction of biochemical parameters after

in vivo LV-FAH delivery. (a) Timeline of NTBC cycling and withdrawal for No. 166 and No.

167 (first row) demonstrated NTBC-independent growth at 98 and 78 days post-treatment. Each gray bar represents one week spent on NTBC. Green line represents when blood was drawn for 
the biochemical data in (b). Timeline of NTBC undertreatment for No. 266, which did not receive LV-FAH and developed HCC, and for No. 158, which did not receive LV-FAH but received appropriate NTBC cycling. (b) Normalization of tyrosine, aspartate aminotransferase (AST), alkaline phosphatase (ALP), ammonia, and total bilirubin levels at 142 days posttreatment. Mean \pm SD are shown for No. 166 and No. 167 alongside historical wild-type and untreated $F A H^{-/-}$controls. P-values are provided for treated animals compared to untreated $F A H^{-/-}$ controls. (c) Age-appropriate decreases and maintenance of minimal alpha-fetoprotein (AFP) levels in No. 166 and No. 167 despite chronic NTBC withdrawal. 
a

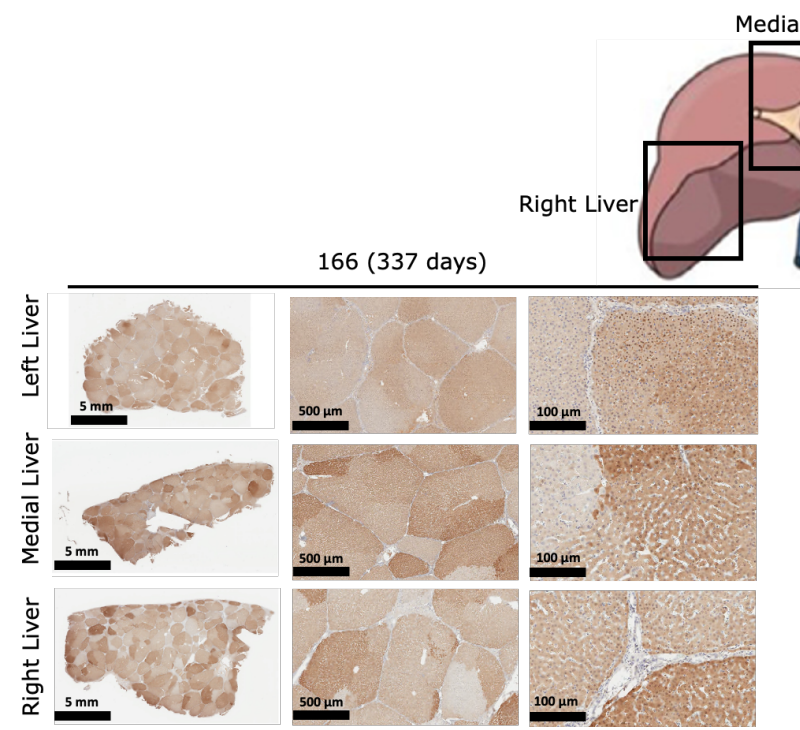

b
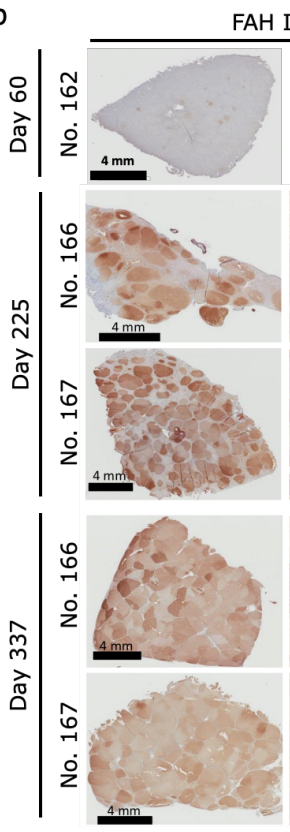

AH IHC
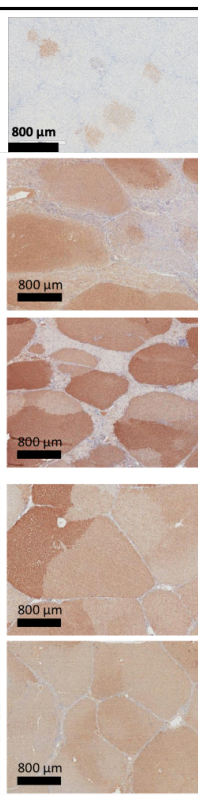

H\&E
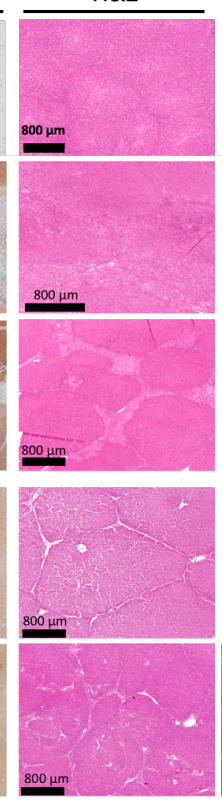

Trichrome
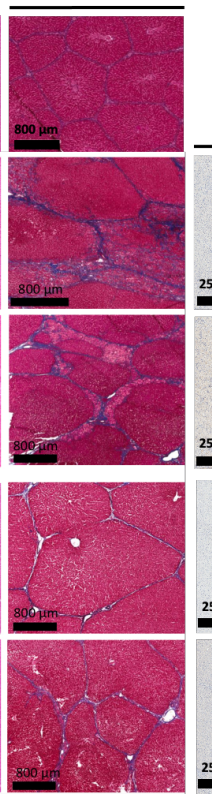

167 (337 days)

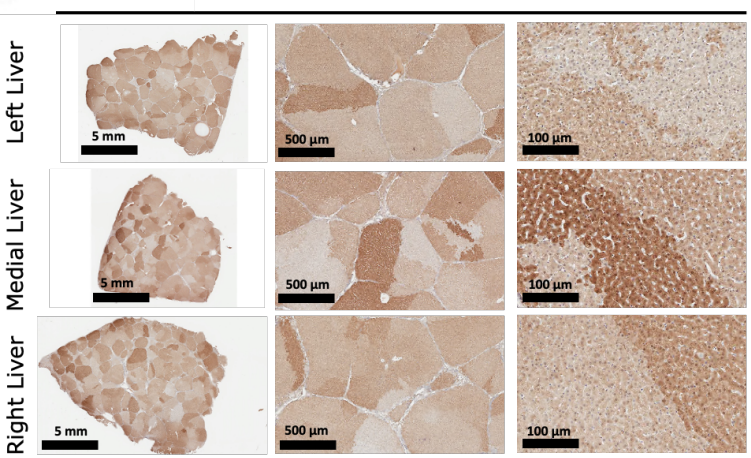

C Wild Type

$\mathrm{HCC}$

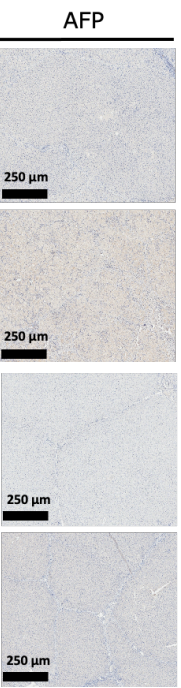

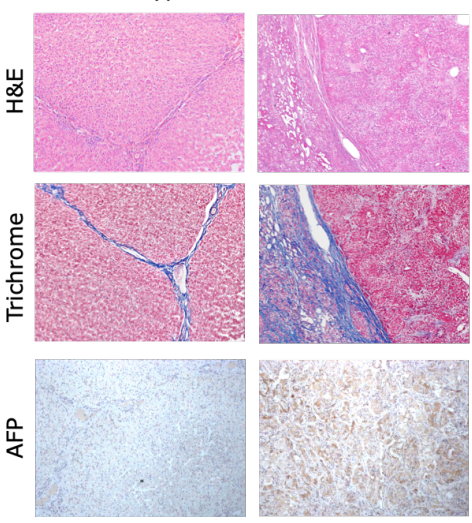

d

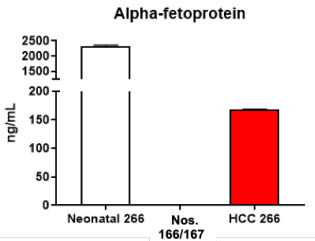

Figure 3. Liver repopulation with FAH-positive hepatocytes after in vivo LV-FAH delivery.

(a) Immunohistochemistry (IHC) for FAH in Nos. 166 and 167 at the time of euthanasia (337

days post-treatment) showing liver repopulation with FAH-positive hepatocytes in all areas of the liver. (b) IHC for FAH in No. 162 at 60 days post-treatment showing multiple FAH-positive nodules in the liver with no presence of significant liver injury on H\&E or Trichrome staining 
(first row). IHC for FAH from laparoscopic liver biopsy in No. 166 (second row) and No. 167 (third row) at 225 days post-treatment showing extensive liver repopulation with FAH-positive hepatocytes, with H\&E and Trichrome staining showing hepatocellular inflammation with moderate fibrosis but no increased AFP staining. IHC for FAH in No. 166 (left medial lobe, fourth row) and No. 167 (left lateral lobe, fifth row) at 337 days post-treatment showing near complete liver repopulation with FAH-positive hepatocytes, with H\&E and Trichrome staining showing resolution of hepatocellular inflammation and fibrosis with no increased AFP staining. (c) H\&E and Trichrome staining of wild type pig liver (left panels) and HCC in an NTBC undertreated $F A H^{-/-}$pig liver (No. 266, right panels) at 1 year show liver fibrosis and cellular alterations in the under-treated animal, while IHC for AFP shows ubiquitous increases in expression consistent with development of HCC in this context. (d) Quantification of AFP in No. 266 shows age-appropriate AFP levels in the neonatal period; however, AFP was slightly elevated at one year, while AFP in Nos. 166 and 167 at 337 days post-treatment was below the limit of quantitation. 

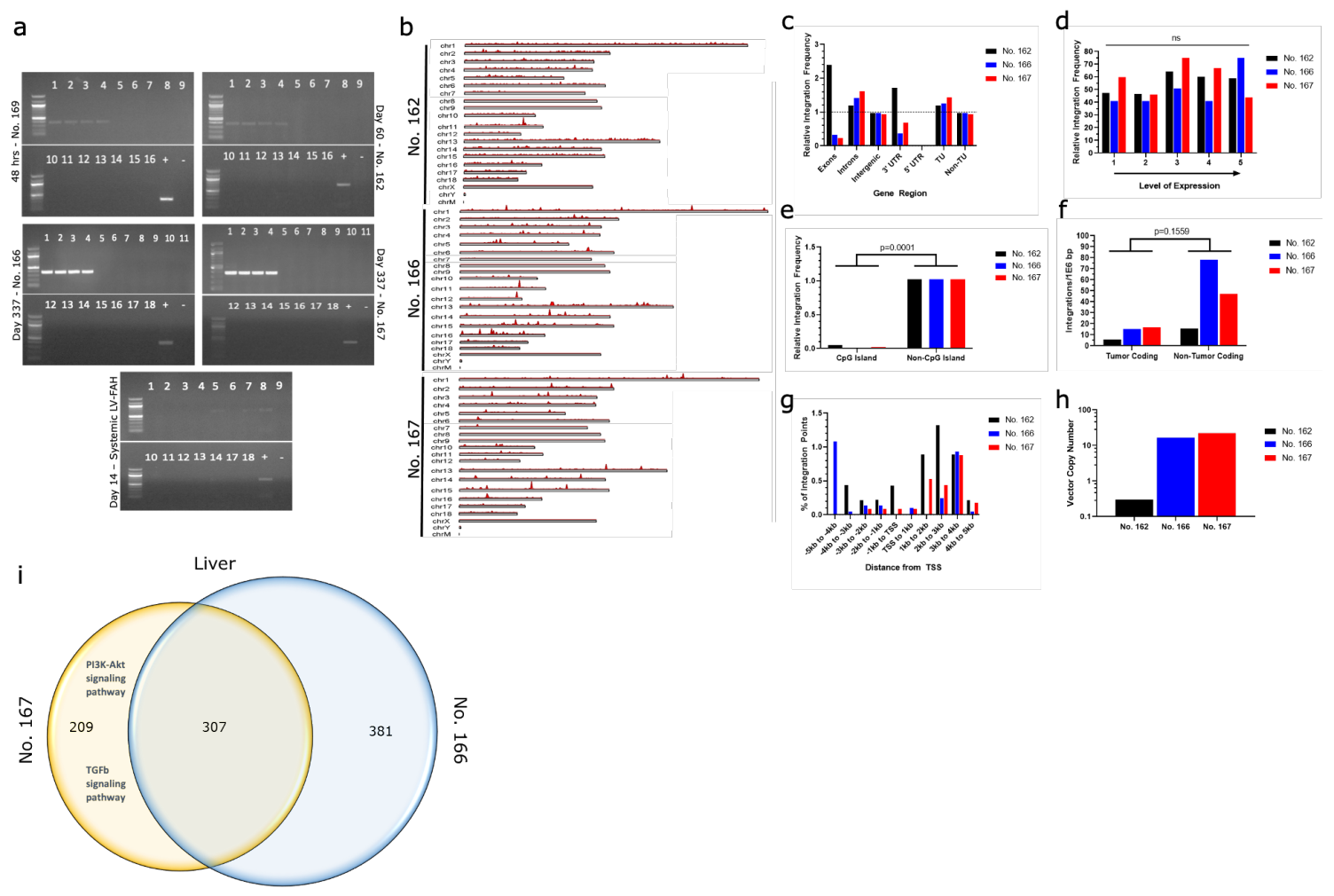

Figure 4. LV-huFAH shows a benign integration profile in pig hepatocytes after in vivo exposure portal vein delivery. (a) Biodistribution of LV-FAH as determined by selective PCR amplification of long terminal repeats over 48 h, 60 days, and 337 days in animals that received portal vein (No. 169, No. 162, No. 166, No. 167, respectively) and systemic injection of LVFAH. Numbers represent the following tissues: 1) right lateral liver, 2) right medial liver, 3) left lateral liver, 4) left medial liver, 5) spleen, 6) kidney, 7) pancreas, 8) duodenum, 9) jejunum, 10) ileum, 11) colon, 12) mesenteric lymph node, 13) heart, 14) right lung, 15) left lung, 16) mediastinal lymph node, 17) testes or ovaries, 18) brain. (b) Chromosomal maps of integration events found in No. 162, No. 166, and No. 167 livers. (c) Relative LV integration across exons, introns, and intragenic regions. (d) Relative LV integration frequency across the genome as stratified by expression level. (e) Relative LV integration frequency showing preference for non- 
CpG island sites, when adjusted for prevalence in the genome. (f) Relative LV integration frequency showing no preference tumor coding genes. (g) Relative integration frequency as stratified by distance from transcription start site. (h) Lentiviral vector copy number found in liver at the time of necropsy. (i) Comparison of LV-FAH integration sites in the livers of No. 167 and No. 166 show high similarity, with 307 common integrations. Gene Set Enrichment Analysis (GSEA) demonstrated no hits in No. 166, but enrichment of the PI3K-Akt and TGFb signaling pathways in No. 167. 

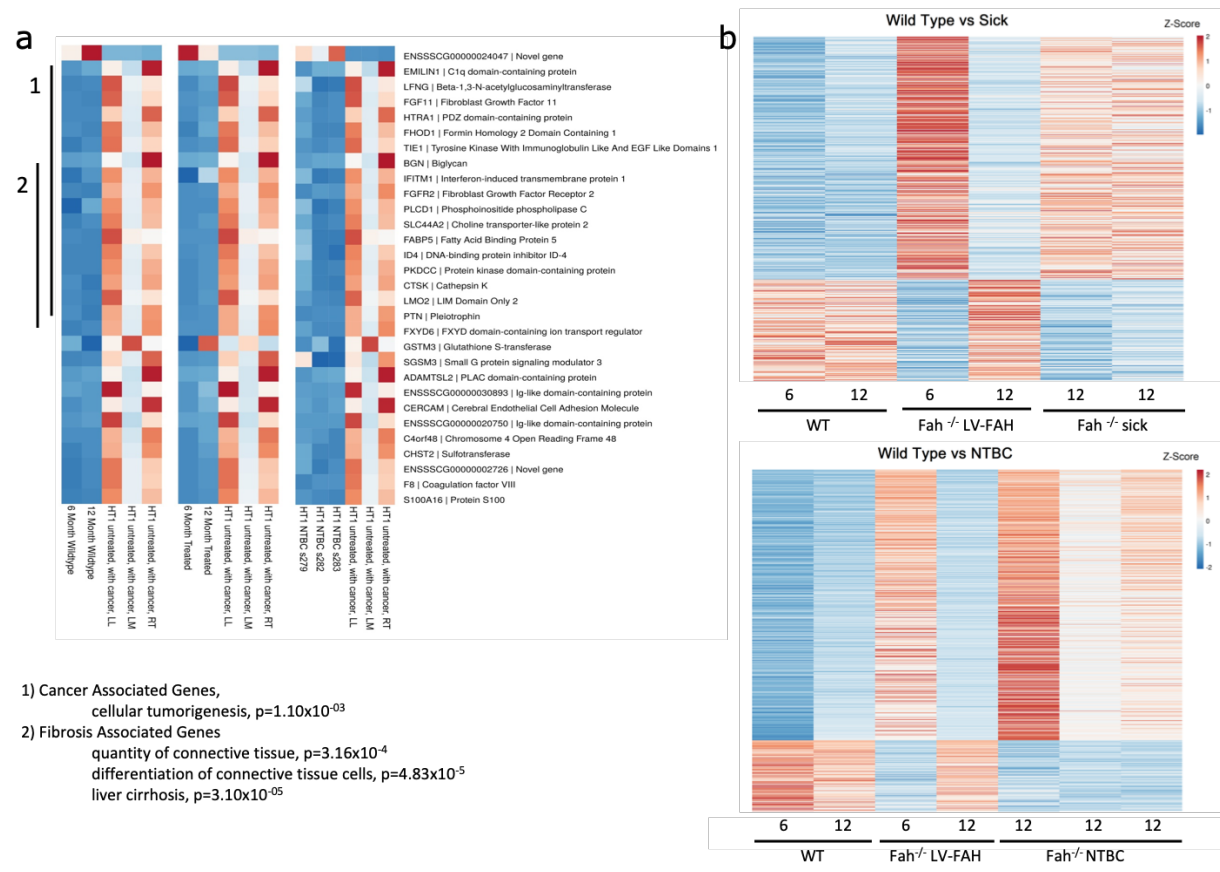

C
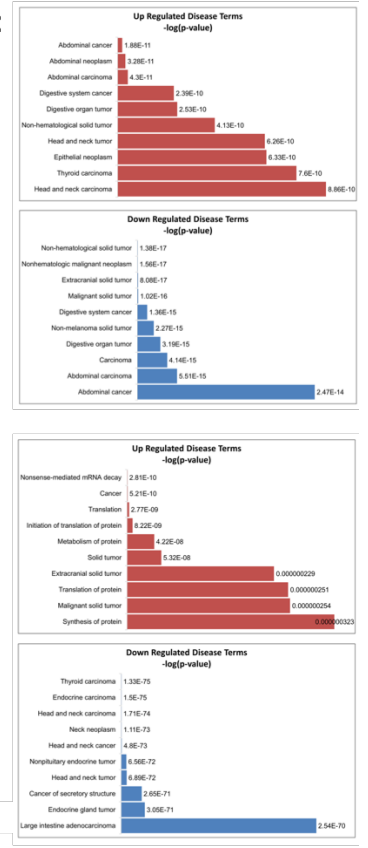

Figure 5. RNA expression analysis shows LV-FAH restores wild type expression profiles

better than standard NTBC treatment. (a) Individual heatmaps comparing RNA expression levels of the genes listed on the right between wildtype pigs at 6 and 12 months of age (left), LVFAH-treated pigs at 6 and 12 months of age (middle) and NTBC-treated pigs at 12 months of age (right). Clusters are identified for 1. cancer- and 2. fibrosis-associated genes. (b) Individual heatmaps showing RNA expression level profiles comparing 6 and 12-month wildtype and LVFAH-treated pigs with either $F A H^{-/-}$NTBC-undertreated pigs (Sick, top) or NTBC-treated pigs (bottom). (c) Gene clusters represented in (b) organized by association with various disease and cancer pathways showing that over the course of 12 months of LV-FAH treatment genes in these pathways are expressed more similarly to wildtype than to the sick or standard NTBC-treated pigs. 
Table 2. Top 10 genes by integration frequency. LV-FAH integration sites were evaluated from representative sections of liver from pigs Nos. 162, 166, and 167. Data are reported by the percent of reads each individual gene represented of the total for that animal/tissue. The number of unique integration sites within a given gene, and the percent of unique integrations sites that represents of the total for each tissue/animal is also presented for context. Finally, the region of highest homology to $F A H$ is presented for each. MGAT4C, included in the top ten genes with highest relative integration frequency for both pigs Nos. 166 and 167, is a transferase essential to production of some $\mathrm{N}$-linked sugar chains.

\begin{tabular}{|c|c|c|c|c|c|c|}
\hline & Gene & $\begin{array}{l}\text { Number of } \\
\text { integration } \\
\text { sites }\end{array}$ & $\begin{array}{l}\text { Percent of } \\
\text { integration } \\
\text { sites }\end{array}$ & $\begin{array}{l}\text { Number of } \\
\text { reads }\end{array}$ & $\begin{array}{l}\text { Percent of } \\
\text { reads }\end{array}$ & $\begin{array}{l}\text { FAH homology } \\
\text { (nucleotides/ } \\
\text { percent) }\end{array}$ \\
\hline \multirow{10}{*}{$\begin{array}{l}\text { No. } \\
162\end{array}$} & ZNF24 & 12 & 1.319 & 29698 & 29.991 & $11 / 100 \%$ \\
\hline & MIR17 & 14 & 1.538 & 17995 & 18.172 & $7 / 100 \%$ \\
\hline & MIR92A-1 & 4 & 0.440 & 4128 & 4.169 & none \\
\hline & TRPC4 & 5 & 0.549 & 3088 & 3.118 & $11 / 100 \%$ \\
\hline & TTR & 3 & 0.330 & 2266 & 2.288 & $12 / 92 \%$ \\
\hline & PRMT6 & 7 & 0.769 & 1954 & 1.973 & $12 / 92 \%$ \\
\hline & HSP90AB1 & 1 & 0.110 & 1663 & 1.679 & $11 / 100 \%$ \\
\hline & FAM107A & 4 & 0.440 & 1290 & 1.303 & $24 / 83 \%$ \\
\hline & NCOA1 & 2 & 0.220 & 1231 & 1.243 & $11 / 100 \%$ \\
\hline & IFI44 & 3 & 0.330 & 1202 & 1.214 & $17 / 82 \%$ \\
\hline \multirow{10}{*}{$\begin{array}{l}\text { No. } \\
166\end{array}$} & ACVR2A & 12 & 0.288 & 468183 & 8.104 & $9 / 100 \%$ \\
\hline & VGLL3 & 46 & 1.103 & 346816 & 6.003 & $10 / 100 \%$ \\
\hline & UOX & 27 & 0.647 & 282277 & 4.886 & $9 / 100 \%$ \\
\hline & MYC & 4 & 0.096 & 240240 & 4.158 & $13 / 92 \%$ \\
\hline & ITGA2 & 98 & 2.350 & 189860 & 3.286 & $11 / 100 \%$ \\
\hline & MIR7135 & 14 & 0.336 & 178740 & 3.094 & $28 / 82 \%$ \\
\hline & ZRANB2 & 77 & 1.847 & 166959 & 2.890 & $39 / 74 \%$ \\
\hline & AKTIP & 13 & 0.312 & 163213 & 2.825 & $11 / 100 \%$ \\
\hline & PNPT1 & 17 & 0.408 & 159257 & 2.757 & $13 / 92 \%$ \\
\hline & MGAT4C & 24 & 0.576 & 100552 & 1.740 & $17 / 88 \%$ \\
\hline \multirow{6}{*}{$\begin{array}{l}\text { No. } \\
167\end{array}$} & DPP4 & 53 & 2.340 & 93939 & 3.649 & $14 / 93 \%$ \\
\hline & ASB3 & 31 & 1.369 & 83777 & 3.255 & $14 / 86 \%$ \\
\hline & SH3GLB1 & 6 & 0.265 & 78216 & 3.038 & $19 / 79 \%$ \\
\hline & DPH5 & 4 & 0.177 & 78209 & 3.038 & $16 / 94 \%$ \\
\hline & RSPO3 & 8 & 0.353 & 77394 & 3.007 & $10 / 100 \%$ \\
\hline & ITGAV & 6 & 0.265 & 75559 & 2.935 & $15 / 87 \%$ \\
\hline
\end{tabular}


bioRxiv preprint doi: https://doi.org/10.1101/2021.01.02.425079; this version posted March 16, 2021. The copyright holder for this preprint (which was not certified by peer review) is the author/funder. All rights reserved. No reuse allowed without permission.

\begin{tabular}{|l|l|l|l|l|l|l|}
\hline & CTSB & 22 & 0.971 & 59593 & 2.315 & $18 / 83 \%$ \\
\cline { 2 - 7 } CCL28 & 50 & 2.208 & 55120 & 2.141 & $11 / 100 \%$ \\
\cline { 2 - 7 } & DDX58 & 21 & 0.927 & 54736 & 2.126 & $21 / 81 \%$ \\
\cline { 2 - 7 } & MGAT4C & 5 & 0.221 & 52320 & 2.032 & $17 / 88 \%$ \\
\hline
\end{tabular}




\section{References}

1. Lindblad, B., Lindstedt, S. \& Steen, G. On the enzymic defects in hereditary tyrosinemia. Proc Natl Acad Sci U S A 74, 4641-4645 (1977).

2. Grompe, M. The pathophysiology and treatment of hereditary tyrosinemia type 1. Semin Liver Dis 21, 563-571 (2001).

3. Endo, F. \& Sun, M.S. Tyrosinaemia type I and apoptosis of hepatocytes and renal tubular cells. J Inherit Metab Dis 25, 227-234 (2002).

4. Lindstedt, S., Holme, E., Lock, E.A., Hjalmarson, O. \& Strandvik, B. Treatment of hereditary tyrosinaemia type I by inhibition of 4-hydroxyphenylpyruvate dioxygenase. Lancet 340, 813-817 (1992).

5. Quebec, N.S.G., et al. The Quebec NTBC Study. Adv Exp Med Biol 959, 187-195 (2017).

6. Chinsky, J.M., et al. Diagnosis and treatment of tyrosinemia type I: a US and Canadian consensus group review and recommendations. Genet Med 19(2017).

7. Almuqbil, M., Knoll, J. \& Chinsky, J.M. Late Development of Hepatocellular Carcinoma in Tyrosinemia Type 1 Despite Nitisinone (NTBC) Treatment. J Pediatr Gastroenterol Nutr 71, e73e75 (2020).

8. van Ginkel, W.G., et al. Long-Term Outcomes and Practical Considerations in the Pharmacological Management of Tyrosinemia Type 1. Paediatr Drugs 21, 413-426 (2019).

9. van Ginkel, W.G., Pennings, J.P. \& van Spronsen, F.J. Liver Cancer in Tyrosinemia Type 1. Adv Exp Med Biol 959, 101-109 (2017).

10. Koelink, C.J., et al. Tyrosinemia type I treated by NTBC: how does AFP predict liver cancer? Mol Genet Metab 89, 310-315 (2006).

11. Holme, E. \& Lindstedt, S. Nontransplant treatment of tyrosinemia. Clin Liver Dis 4, 805-814 (2000).

12. Walker, H., Pitkanen, M., Rahman, Y. \& Barrington, S.F. Three Cases of Hereditary Tyrosinaemia Type 1: Neuropsychiatric Outcomes and Brain Imaging Following Treatment with NTBC. JIMD Rep 40, 97-103 (2018).

13. van Ginkel, W.G., Jahja, R., Huijbregts, S.C.J. \& van Spronsen, F.J. Neurological and Neuropsychological Problems in Tyrosinemia Type I Patients. Adv Exp Med Biol 959, 111-122 (2017).

14. Thimm, E., et al. Increase of CSF tyrosine and impaired serotonin turnover in tyrosinemia type I. Mol Genet Metab 102, 122-125 (2011).

15. Garcia, M.I., de la Parra, A., Arias, C., Arredondo, M. \& Cabello, J.F. Long-term cognitive functioning in individuals with tyrosinemia type 1 treated with nitisinone and protein-restricted diet. Mol Genet Metab Rep 11, 12-16 (2017).

16. De Laet, C., et al. Neuropsychological outcome of NTBC-treated patients with tyrosinaemia type 1. Dev Med Child Neurol 53, 962-964 (2011).

17. Ellaway, C.J., et al. Outcome of tyrosinaemia type III. J Inherit Metab Dis 24, 824-832 (2001).

18. Heylen, E., et al. Tyrosinemia Type III detected via neonatal screening: management and outcome. Mol Genet Metab 107, 605-607 (2012).

19. Barroso, F., et al. Tyrosinemia Type lii: A Case Report of Siblings and Literature Review. Rev Paul Pediatr 38, e2018158 (2020).

20. Cerone, R., et al. Tyrosinemia type III: diagnosis and ten-year follow-up. Acta Paediatr 86, 10131015 (1997).

21. van Ginkel, W.G., et al. Neurocognitive outcome in tyrosinemia type 1 patients compared to healthy controls. Orphanet J Rare Dis 11, 87 (2016). 
22. Thimm, E., et al. Neurocognitive outcome in patients with hypertyrosinemia type I after longterm treatment with NTBC. J Inherit Metab Dis 35, 263-268 (2012).

23. Bendadi, F., et al. Impaired cognitive functioning in patients with tyrosinemia type I receiving nitisinone. J Pediatr 164, 398-401 (2014).

24. Mayorandan, S., et al. Cross-sectional study of 168 patients with hepatorenal tyrosinaemia and implications for clinical practice. Orphanet J Rare Dis 9, 107 (2014).

25. Pohorecka, M., et al. Behavioral and intellectual functioning in patients with tyrosinemia type $\mathrm{I}$. Pediatr Endocrinol Diabetes Metab 18, 96-100 (2012).

26. Masurel-Paulet, A., et al. NTBC treatment in tyrosinaemia type I: long-term outcome in French patients. J Inherit Metab Dis 31, 81-87 (2008).

27. Couce, M.L., et al. Evolution of tyrosinemia type 1 disease in patients treated with nitisinone in Spain. Medicine (Baltimore) 98, e17303 (2019).

28. Raimann, E., et al. [Clinical follow up of Chilean patients with tyrosinemia type 1 treated with 2(2-nitro-4-trifluoromethylbenzoyl)-1,3-ciclohexanedione (NTBC)]. Rev Med Chil 140, 169-175 (2012).

29. Colemonts-Vroninks, H., et al. Oxidative Stress, Glutathione Metabolism, and Liver Regeneration Pathways Are Activated in Hereditary Tyrosinemia Type 1 Mice upon Short-Term Nitisinone Discontinuation. Genes (Basel) 12(2020).

30. Hickey, R.D., et al. Curative ex vivo liver-directed gene therapy in a pig model of hereditary tyrosinemia type 1. Sci Transl Med 8, 349ra399 (2016).

31. Paulk, N.K., et al. Adeno-associated virus gene repair corrects a mouse model of hereditary tyrosinemia in vivo. Hepatology 51, 1200-1208 (2010).

32. Overturf, K., et al. Adenovirus-mediated gene therapy in a mouse model of hereditary tyrosinemia type I. Hum Gene Ther 8, 513-521 (1997).

33. Overturf, K., et al. Hepatocytes corrected by gene therapy are selected in vivo in a murine model of hereditary tyrosinaemia type I. Nat Genet 12, 266-273 (1996).

34. Yin, H., et al. Genome editing with Cas9 in adult mice corrects a disease mutation and phenotype. Nat Biotechnol 32, 551-553 (2014).

35. Pankowicz, F.P., et al. Reprogramming metabolic pathways in vivo with CRISPR/Cas9 genome editing to treat hereditary tyrosinaemia. Nat Commun 7, 12642 (2016).

36. Grisham, J.W. Interspecies comparison of liver carcinogenesis: implications for cancer risk assessment. Carcinogenesis 18, 59-81 (1997).

37. Al-Dhalimy, M., Overturf, K., Finegold, M. \& Grompe, M. Long-term therapy with NTBC and tyrosine-restricted diet in a murine model of hereditary tyrosinemia type I. Mol Genet Metab 75, 38-45 (2002).

38. Holliday, R. Neoplastic transformation: the contrasting stability of human and mouse cells. Cancer Surv 28, 103-115 (1996).

39. Rangarajan, A. \& Weinberg, R.A. Opinion: Comparative biology of mouse versus human cells: modelling human cancer in mice. Nat Rev Cancer 3, 952-959 (2003).

40. Nathwani, A.C., et al. Adenovirus-associated virus vector-mediated gene transfer in hemophilia B. N Engl J Med 365, 2357-2365 (2011).

41. Wang, D., et al. Adenovirus-Mediated Somatic Genome Editing of Pten by CRISPR/Cas9 in Mouse Liver in Spite of Cas9-Specific Immune Responses. Hum Gene Ther 26, 432-442 (2015).

42. Hu, C., Busuttil, R.W. \& Lipshutz, G.S. RH10 provides superior transgene expression in mice when compared with natural AAV serotypes for neonatal gene therapy. J Gene Med 12, 766-778 (2010). 
43. Tai, D.S., Hu, C., Kim, E.H. \& Lipshutz, G.S. Augmentation of transgene-encoded protein after neonatal injection of adeno-associated virus improves hepatic copy number without immune responses. Pediatr Res 78, 239-246 (2015).

44. Colella, P., et al. AAV Gene Transfer with Tandem Promoter Design Prevents Anti-transgene Immunity and Provides Persistent Efficacy in Neonate Pompe Mice. Mol Ther Methods Clin Dev 12, 85-101 (2019).

45. Follenzi, A., Santambrogio, L. \& Annoni, A. Immune responses to lentiviral vectors. Curr Gene Ther 7, 306-315 (2007).

46. al Yacoub, N., Romanowska, M., Haritonova, N. \& Foerster, J. Optimized production and concentration of lentiviral vectors containing large inserts. J Gene Med 9, 579-584 (2007).

47. Binny, C., et al. AAV-mediated gene transfer in the perinatal period results in expression of FVII at levels that protect against fatal spontaneous hemorrhage. Blood 119, 957-966 (2012).

48. Cantore, A., et al. Liver-directed lentiviral gene therapy in a dog model of hemophilia B. Sci Transl Med 7, 277ra228 (2015).

49. Liu, S.K., et al. Hypertrophic cardiomyopathy in pigs: quantitative pathologic features in 55 cases. Cardiovasc Pathol 3, 261-268 (1994).

50. Huang, S.Y., et al. Heritability estimate of hypertrophic cardiomyopathy in pigs (Sus scrofa domestica). Lab Anim Sci 46, 310-314 (1996).

51. Bell, J.M. A study of rates of growth of Yorkshire, Lacombe, Landrace, and Crossbred pigs from birth to $200 \mathrm{lb}$. Can J Animal Sci 44, 315-319 (1964).

52. Birteeb, P.T., Tetteh, S.O. \& Salifu, A.S. Growth Performance and Weight Estimation of Large White Piglets Weaned at Different Ages. Research \& Reviews: Journal of Veterinary Science and Technology 4, 15-23 (2015).

53. Ingvarsson, B.I., Carlsson, R.N. \& Karlsson, B.W. Synthesis of alpha-fetoprotein, albumin and total serum protein in neonatal pigs. Biol Neonate 34, 259-268 (1978).

54. Bedossa, P. \& Poynard, T. An algorithm for the grading of activity in chronic hepatitis C. The METAVIR Cooperative Study Group. Hepatology 24, 289-293 (1996).

55. Brown, B.D., et al. In vivo administration of lentiviral vectors triggers a type I interferon response that restricts hepatocyte gene transfer and promotes vector clearance. Blood 109, 2797-2805 (2007).

56. Zhao, Y., Stepto, H. \& Schneider, C.K. Development of the First World Health Organization Lentiviral Vector Standard: Toward the Production Control and Standardization of LentivirusBased Gene Therapy Products. Hum Gene Ther Methods 28, 205-214 (2017).

57. Nicolas, C.T., et al. Hepatocyte spheroids as an alternative to single cells for transplantation after ex vivo gene therapy in mice and pig models. Surgery 164, 473-481 (2018).

58. Zhang, H.E., Henderson, J.M. \& Gorrell, M.D. Animal models for hepatocellular carcinoma. Biochim Biophys Acta Mol Basis Dis 1865, 993-1002 (2019).

59. Macek Jilkova, Z., Kurma, K. \& Decaens, T. Animal Models of Hepatocellular Carcinoma: The Role of Immune System and Tumor Microenvironment. Cancers (Basel) 11(2019).

60. Schachtschneider, K.M., et al. A validated, transitional and translational porcine model of hepatocellular carcinoma. Oncotarget 8, 63620-63634 (2017).

61. Schachtschneider, K.M., et al. The Oncopig Cancer Model: An Innovative Large Animal Translational Oncology Platform. Front Oncol 7, 190 (2017).

62. Desai, A., Sandhu, S., Lai, J.P. \& Sandhu, D.S. Hepatocellular carcinoma in non-cirrhotic liver: A comprehensive review. World J Hepatol 11, 1-18 (2019).

63. Kienstra, N.S., et al. Daily variation of NTBC and its relation to succinylacetone in tyrosinemia type 1 patients comparing a single dose to two doses a day. J Inherit Metab Dis 41, 181-186 (2018). 
64. Schroder, A.R., et al. HIV-1 integration in the human genome favors active genes and local hotspots. Cell 110, 521-529 (2002).

65. Montini, E., et al. The genotoxic potential of retroviral vectors is strongly modulated by vector design and integration site selection in a mouse model of HSC gene therapy. J Clin Invest 119, 964-975 (2009).

66. Kaiser, R.A., et al. Hepatotoxicity and Toxicology of In Vivo Lentiviral Vector Administration in Healthy and Liver-Injury Mouse Models. Hum Gene Ther Clin Dev 30, 57-66 (2019).

67. Ranzani, M., et al. Lentiviral vector-based insertional mutagenesis identifies genes associated with liver cancer. Nat Methods 10, 155-161 (2013).

68. Themis, M., et al. Oncogenesis following delivery of a nonprimate lentiviral gene therapy vector to fetal and neonatal mice. Mol Ther 12, 763-771 (2005).

69. Papanikolaou, E., et al. Cell cycle status of CD34(+) hemopoietic stem cells determines lentiviral integration in actively transcribed and development-related genes. Mol Ther 23, 683-696 (2015).

70. Niu, Z.S., Niu, X.J. \& Wang, W.H. Genetic alterations in hepatocellular carcinoma: An update. World J Gastroenterol 22, 9069-9095 (2016).

71. Seda Neto, J., et al. HCC prevalence and histopathological findings in liver explants of patients with hereditary tyrosinemia type 1. Pediatr Blood Cancer 61, 1584-1589 (2014).

72. van Spronsen, F.J., Bijleveld, C.M., van Maldegem, B.T. \& Wijburg, F.A. Hepatocellular carcinoma in hereditary tyrosinemia type I despite 2-(2 nitro-4-3 trifluoro- methylbenzoyl)-1, 3cyclohexanedione treatment. J Pediatr Gastroenterol Nutr 40, 90-93 (2005).

73. Hacein-Bey-Abina, S., et al. Insertional oncogenesis in 4 patients after retrovirus-mediated gene therapy of SCID-X1. J Clin Invest 118, 3132-3142 (2008).

74. Aiuti, A., et al. Lentiviral hematopoietic stem cell gene therapy in patients with Wiskott-Aldrich syndrome. Science 341, 1233151 (2013).

75. Biffi, A., et al. Lentiviral hematopoietic stem cell gene therapy benefits metachromatic leukodystrophy. Science 341, 1233158 (2013).

76. David, R.M. \& Doherty, A.T. Viral Vectors: The Road to Reducing Genotoxicity. Toxicol Sci 155, 315-325 (2017).

77. Vranckx, L.S., Demeulemeester, J., Debyser, Z. \& Gijsbers, R. Towards a Safer, More Randomized Lentiviral Vector Integration Profile Exploring Artificial LEDGF Chimeras. PLoS One 11, e0164167 (2016).

78. Sheu, J., et al. Large-scale production of lentiviral vector in a closed system hollow fiber bioreactor. Mol Ther Methods Clin Dev 2, 15020 (2015).

79. Bauler, M., et al. Production of Lentiviral Vectors Using Suspension Cells Grown in Serum-free Media. Mol Ther Methods Clin Dev 17, 58-68 (2020).

80. Schiedner, G., et al. Selective depletion or blockade of Kupffer cells leads to enhanced and prolonged hepatic transgene expression using high-capacity adenoviral vectors. Mol Ther 7, 3543 (2003).

81. Hickey, R.D., et al. Efficient production of Fah-null heterozygote pigs by chimeric adenoassociated virus-mediated gene knockout and somatic cell nuclear transfer. Hepatology 54, 1351-1359 (2011).

82. Hickey, R.D., et al. Fumarylacetoacetate hydrolase deficient pigs are a novel large animal model of metabolic liver disease. Stem Cell Res 13, 144-153 (2014).

83. Wang, X., et al. Kinetics of liver repopulation after bone marrow transplantation. Am J Pathol 161, 565-574 (2002).

84. Martin, M. Cutadapt removes adapter sequences from high-throughput sequencing reads. 2011 17, 3 (2011). 
85. Li, H. \& Durbin, R. Fast and accurate short read alignment with Burrows-Wheeler transform. Bioinformatics 25, 1754-1760 (2009).

86. Wang, J., Vasaikar, S., Shi, Z., Greer, M. \& Zhang, B. WebGestalt 2017: a more comprehensive, powerful, flexible and interactive gene set enrichment analysis toolkit. Nucleic Acids Res $\mathbf{4 5}$, W130-W137 (2017).

87. Dobin, A., et al. STAR: ultrafast universal RNA-seq aligner. Bioinformatics 29, 15-21 (2013).

88. Liao, Y., Smyth, G.K. \& Shi, W. featureCounts: an efficient general purpose program for assigning sequence reads to genomic features. Bioinformatics 30, 923-930 (2014).

89. Robinson, M.D., McCarthy, D.J. \& Smyth, G.K. edgeR: a Bioconductor package for differential expression analysis of digital gene expression data. Bioinformatics 26, 139-140 (2010).

90. Kramer, A., Green, J., Pollard, J., Jr. \& Tugendreich, S. Causal analysis approaches in Ingenuity Pathway Analysis. Bioinformatics 30, 523-530 (2014).

91. Metsalu, T. \& Vilo, J. ClustVis: a web tool for visualizing clustering of multivariate data using Principal Component Analysis and heatmap. Nucleic Acids Res 43, W566-570 (2015). 


\section{Supplemental Data}

Supplemental Figure 1. Further acute inflammatory response (IL-8 and platelets) after systemic LV-FAH administration compared to reference portal vein LV-GFP delivery (a) and further vital signs (temperature) after portal LV-FAH administration (b).

a

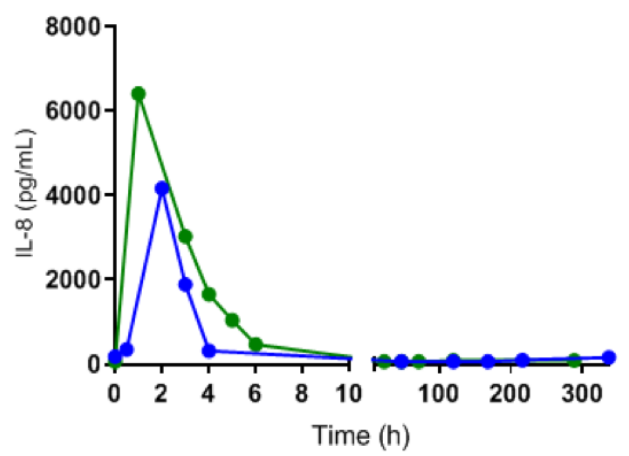

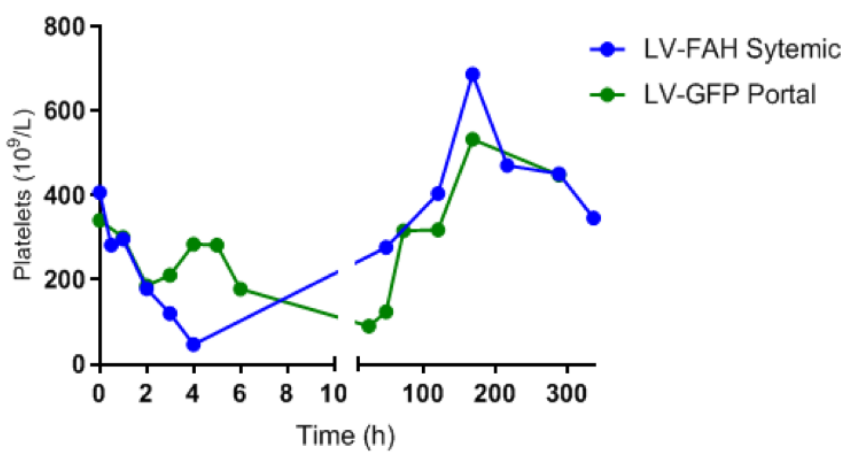

b

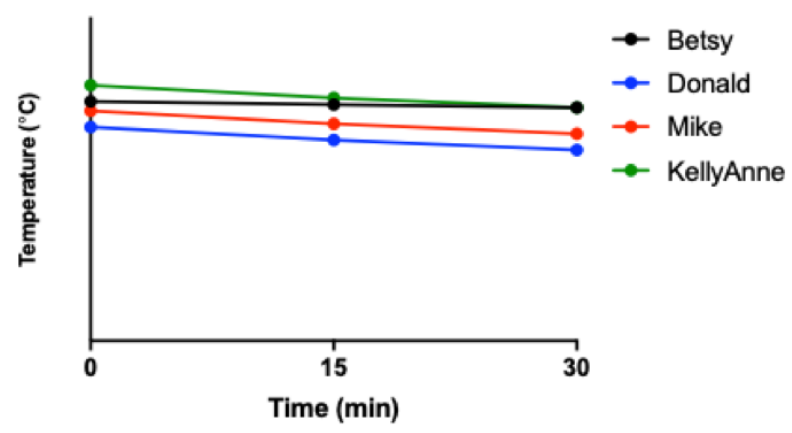


Supplemental Figure 2. H\&E staining of myocardium showing myocyte hypertrophy and disarray characteristic of hypertrophic cardiomyopathy in pig No. 169 (a) as compared to $\mathrm{FAH}^{-/-}$age-matched control (b).
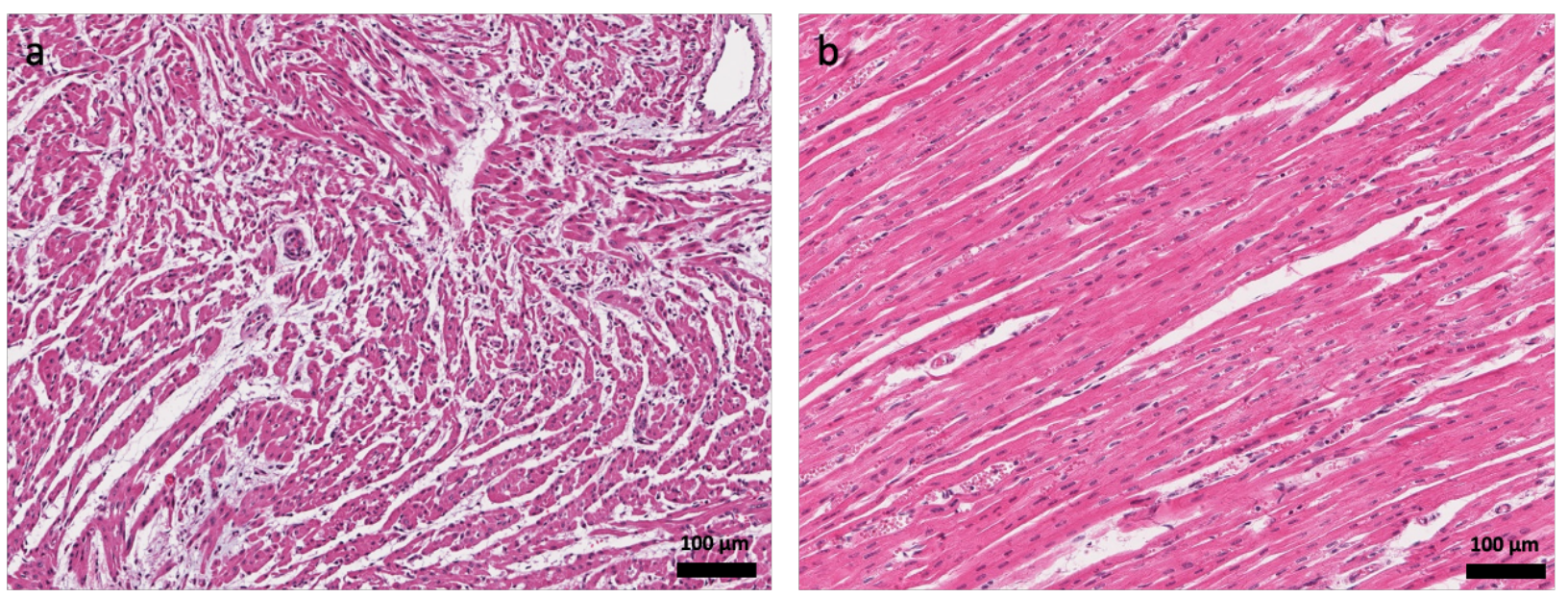
Supplemental Figure 3. Tyrosine levels over time. Day 0 represents pre-treatment blood draw. Day 337 represents post-mortem collection.

No. 166

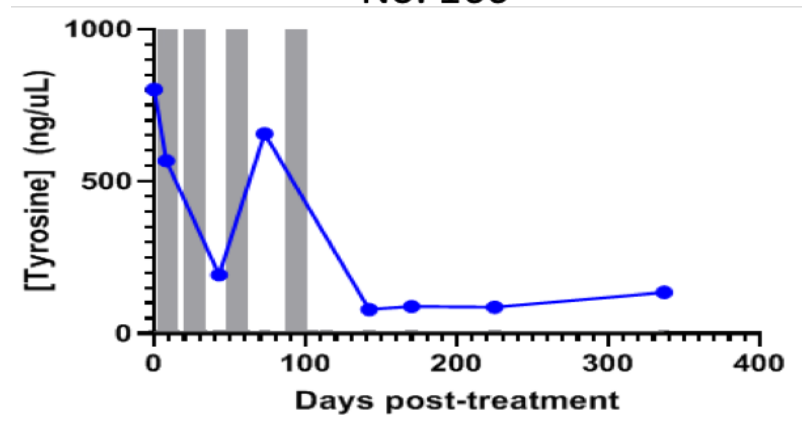

No. 167

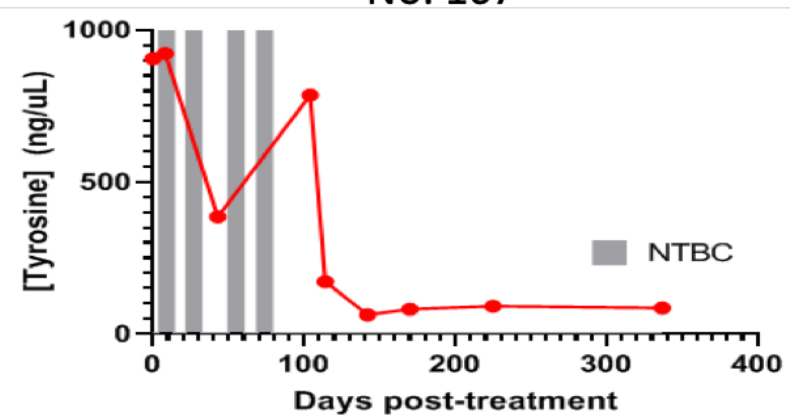

Supplemental Table 1. Summary of blood sample analyses for long term LV-FAH dosed animals. Day 0 represents pre-treatment blood draw. Day 337 represents post-mortem collection.

\begin{tabular}{|l|l|llllllllll|}
\hline Days post-treatment & $\mathbf{0}$ & $\mathbf{8}$ & $\mathbf{4 3}$ & $\mathbf{7 3}$ & $\mathbf{1 0 4}$ & $\mathbf{1 1 4}$ & $\mathbf{1 4 2}$ & $\mathbf{1 7 0}$ & $\mathbf{2 2 5}$ & $\mathbf{3 3 7}$ \\
\hline No. 166 & ALB & 3.1 & 3 & 2.3 & 2.7 & - & - & 3.2 & 3.3 & 3.2 & 4.2 \\
& ALP & 372 & 321 & 551 & 298 & - & - & 195 & 180 & 120 & 68 \\
& ALT & 86 & 57 & 99 & 81 & - & - & 68 & 65 & 48 & 32 \\
& AST & 74 & 44 & 37 & 31 & - & - & 44 & 25 & 19 & 40 \\
& BILD & $<0.1$ & $<0.1$ & $<0.1$ & $<0.1$ & - & - & $<0.1$ & $<0.1$ & $<0.1$ & $<0.1$ \\
& BILT & $<0.2$ & $<0.2$ & $<0.2$ & $<0.2$ & - & - & $<0.2$ & $<0.2$ & $<0.2$ & $<0.2$ \\
& GGT & 83 & 126 & 126 & 106 & - & - & 166 & 89 & 66 & 81 \\
& Ammonia & 23 & 16 & 49 & 28 & - & - & 23 & 19 & 59 & 301 \\
& PT & Clot & 10.2 & 11.7 & 11 & - & - & 11.2 & 10.7 & 11.1 & - \\
& INR & Clot & 0.9 & 1.1 & 1 & - & - & 1 & 1 & 1 & - \\
& Phe & 57 & 44 & 91 & 74 & - & - & 68 & 83 & 116 & 203 \\
& Tyr & 802 & 568 & 193 & 656 & - & - & 79 & 89 & 86 & 135 \\
\hline No. 167 & ALB & 3.7 & 3.6 & 3.2 & - & 2.9 & 3.5 & 3.7 & 3.7 & 4 & 3.3 \\
& ALP & 307 & 216 & 514 & - & 170 & 174 & 135 & 104 & 61 & 24 \\
& ALT & 71 & 72 & 65 & - & 67 & 56 & 55 & 40 & 38 & 32 \\
& AST & Hem & 71 & 68 & - & 23 & 58 & 35 & 16 & 20 & 81 \\
& BILD & $<0.1$ & $<0.1$ & $<0.1$ & - & $<0.1$ & $<0.1$ & $<0.1$ & $<0.1$ & $<0.1$ & $<0.1$ \\
& BILT & $<0.2$ & $<0.2$ & $<0.2$ & - & $<0.2$ & $<0.2$ & $<0.2$ & $<0.2$ & $<0.2$ & $<0.2$ \\
& GGT & 70 & 136 & 204 & - & 82 & 84 & 82 & 63 & 56 & 125 \\
& Ammonia & 16 & 15 & 33 & - & $<10$ & 11 & $<10$ & 10 & 38 & 207 \\
& PT & 11.1 & 10.3 & 11.7 & - & Clot & 10.4 & 11.8 & 11.1 & 11.7 & 9.1 \\
& INR & 1 & 0.9 & 1.1 & - & Clot & 0.9 & 1.1 & 1 & 1.1 & 0.8 \\
& Phe & 40 & 38 & 121 & - & 45 & 55 & 57 & 72 & 92 & 140 \\
& Tyr & 905 & 923 & 385 & - & 786 & 172 & 63 & 81 & 91 & 85 \\
\hline
\end{tabular}

“-” indicates sample not tested for that animal occasion. 
Supplemental Figure 4. IHC for FAH, H\&E, and Trichrome staining in No. 169 at $48 \mathrm{~h}$ post-treatment.
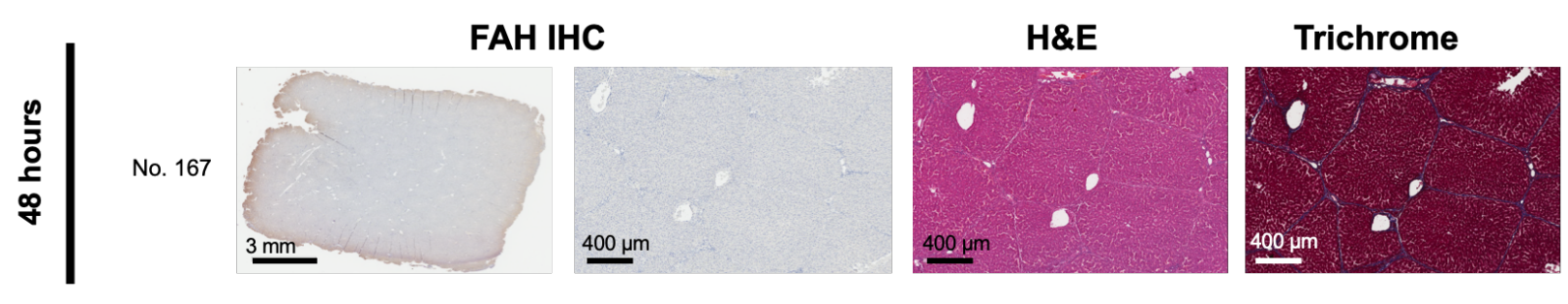

Supplemental Figure 5. H\&E staining at high magnification showing restoration of normal hepatic microarchitecture in LV-FAH treated pig (a) as compared to healthy wild-type (b) and $\mathrm{FAH}^{-/-}$sick (c) pigs. Gross liver sections from $\mathrm{LV}$-FAH treated pig at the time of euthanasia showing no macroscopically visible adenomas (d).
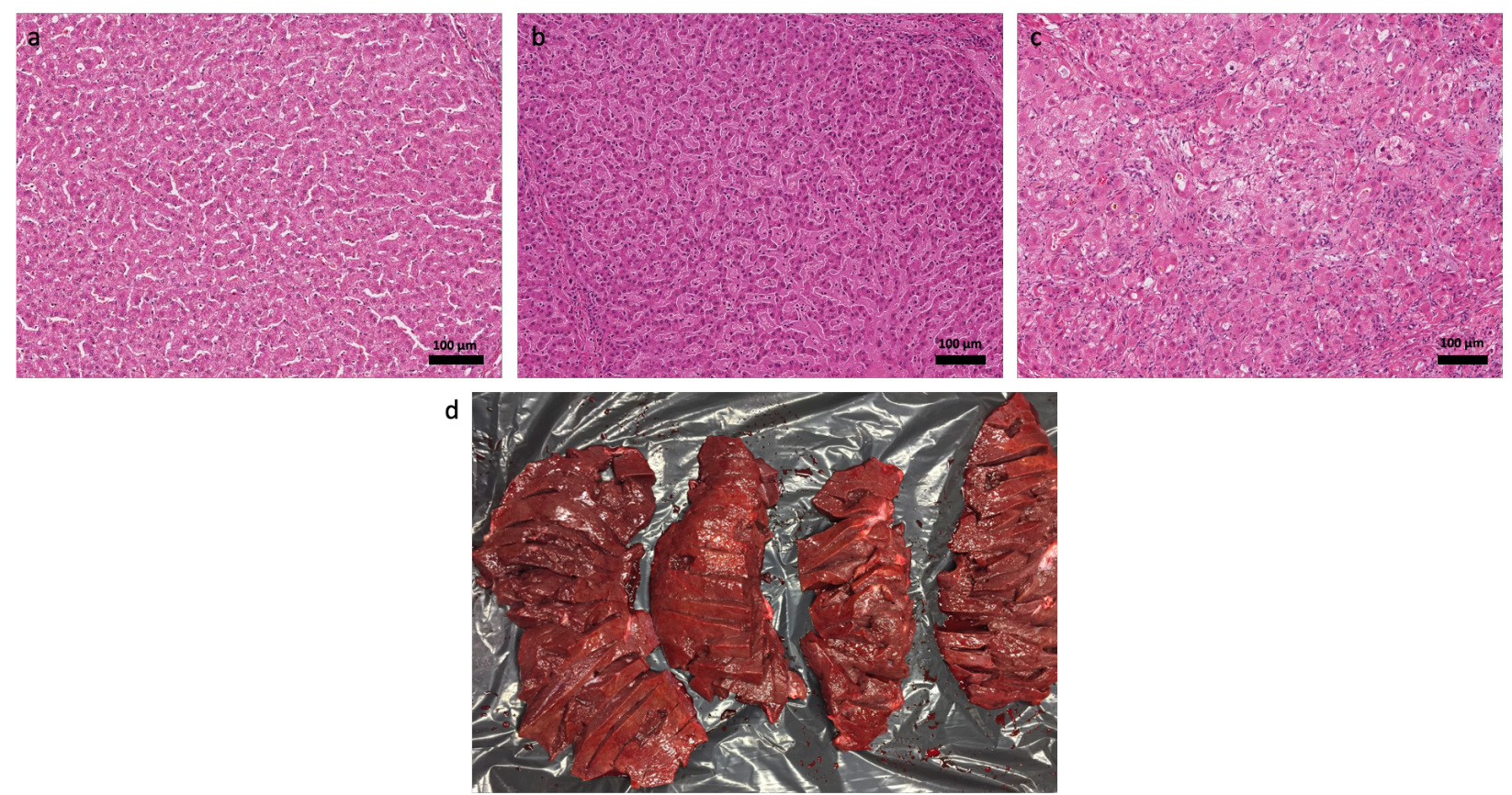
Supplemental Table 2. Percent of fibrotic liver sections per independent pathologist review.

\begin{tabular}{|c|c|c|c|c|}
\hline Pig & Time & Site & Sample & $\begin{array}{l}\% \\
\text { abnormal }\end{array}$ \\
\hline \multirow[t]{18}{*}{16166 (No. 166) } & \multirow[t]{2}{*}{ Day 142} & \multicolumn{2}{|l|}{ Biopsy 1} & 60 \\
\hline & & \multicolumn{2}{|l|}{ Biopsy 2} & 100 \\
\hline & \multirow[t]{16}{*}{ Day 337} & \multirow[t]{4}{*}{ Right Lateral } & Anterior Superior & 20 \\
\hline & & & Posterior Superior & 20 \\
\hline & & & Anterior Inferior & 30 \\
\hline & & & Posterior Inferior & 30 \\
\hline & & \multirow[t]{4}{*}{ Right Medial } & Anterior Superior & 10 \\
\hline & & & Posterior Superior & 10 \\
\hline & & & Anterior Inferior & 20 \\
\hline & & & Posterior Inferior & 5 \\
\hline & & \multirow[t]{4}{*}{ Left Medial } & Anterior Superior & 2 \\
\hline & & & Posterior Superior & 5 \\
\hline & & & Anterior Inferior & 20 \\
\hline & & & Posterior Inferior & 30 \\
\hline & & \multirow[t]{4}{*}{ Left Lateral } & Anterior Superior & 0 \\
\hline & & & Posterior Superior & 20 \\
\hline & & & Anterior Inferior & 10 \\
\hline & & & Posterior Inferior & 5 \\
\hline \multirow[t]{18}{*}{ 16P176 (No. 167) } & \multirow[t]{2}{*}{ Day 142} & \multicolumn{2}{|l|}{ Biopsy 1} & 50 \\
\hline & & \multicolumn{2}{|l|}{ Biopsy 2} & 50 \\
\hline & \multirow[t]{16}{*}{ Day 337} & \multirow[t]{4}{*}{ Right Lateral } & Anterior Superior & 20 \\
\hline & & & Posterior Superior & 5 \\
\hline & & & Anterior Inferior & 10 \\
\hline & & & Posterior Inferior & 20 \\
\hline & & \multirow[t]{4}{*}{ Right Medial } & Anterior Superior & 2 \\
\hline & & & Posterior Superior & 10 \\
\hline & & & Anterior Inferior & 5 \\
\hline & & & Posterior Inferior & 30 \\
\hline & & \multirow[t]{4}{*}{ Left Medial } & Anterior Superior & 2 \\
\hline & & & Posterior Superior & 5 \\
\hline & & & Anterior Inferior & 20 \\
\hline & & & Posterior Inferior & 10 \\
\hline & & \multirow[t]{4}{*}{ Left Lateral } & Anterior Superior & 2 \\
\hline & & & Posterior Superior & 5 \\
\hline & & & Anterior Inferior & 2 \\
\hline & & & Posterior Inferior & 20 \\
\hline
\end{tabular}


Supplemental Table 3. LV-FAH integration mapping statistics.

\begin{tabular}{|c|c|c|c|c|}
\hline $\begin{array}{c}\text { Percent of } \\
\text { Reads with } \\
\text { LV-FAH }\end{array}$ & $\begin{array}{c}\text { Total Used Reads } \\
\text { for Mapping }\end{array}$ & $\begin{array}{c}\text { Mapped Reads } \\
\text { (Total) }\end{array}$ & $\begin{array}{c}\text { Integration } \\
\text { Points at } \\
\text { >= 1X }\end{array}$ & $\begin{array}{c}\text { Integration } \\
\text { Points at } \\
\text { >=5X }\end{array}$ \\
\hline 38.44 & $55,968,431$ & $637,573(1.14 \%)$ & 17,906 & 4,425 \\
\hline 14.21 & $66,139,096$ & $14,278,183(21.59 \%)$ & 39,085 & 8,642 \\
\hline 28.54 & $53,105,410$ & $7,848,599(14.78 \%)$ & 35,298 & 6,330 \\
\hline
\end{tabular}

